\title{
Direct numerical simulations of forced and unforced separation bubbles on an airfoil at incidence
}

\author{
L. E. JONES, R. D. SANDBERG AND N. D. SANDHAM \\ Aerodynamics and Flight Mechanics Research Group, School of Engineering Sciences, University of \\ Southampton, Southampton, SO17 1BJ, UK
}

(Received 21 August 2007 and in revised form 23 January 2008)

Direct numerical simulations (DNS) of laminar separation bubbles on a NACA-0012 airfoil at $R_{c}=5 \times 10^{4}$ and incidence $5^{\circ}$ are presented. Initially volume forcing is introduced in order to promote transition to turbulence. After obtaining sufficient data from this forced case, the explicitly added disturbances are removed and the simulation run further. With no forcing the turbulence is observed to self-sustain, with increased turbulence intensity in the reattachment region. A comparison of the forced and unforced cases shows that the forcing improves the aerodynamic performance whilst requiring little energy input. Classical linear stability analysis is performed upon the time-averaged flow field; however no absolute instability is observed that could explain the presence of self-sustaining turbulence. Finally, a series of simplified DNS are presented that illustrate a three-dimensional absolute instability of the twodimensional vortex shedding that occurs naturally. Three-dimensional perturbations are amplified in the braid region of developing vortices, and subsequently convected upstream by local regions of reverse flow, within which the upstream velocity magnitude greatly exceeds that of the time-average. The perturbations are convected into the braid region of the next developing vortex, where they are amplified further, hence the cycle repeats with increasing amplitude. The fact that this transition process is independent of upstream disturbances has implications for modelling separation bubbles.

\section{Introduction}

Under an adverse pressure gradient a boundary layer may separate, leading to reverse (upstream) fluid flow. Within the separated region disturbances are strongly amplified, typically leading to transition to turbulence. The resultant turbulent flow enhances mixing and momentum transfer in the wall-normal direction, and causes the boundary layer to reattach. This system of laminar separation, transition and turbulent reattachment is referred to as a laminar (or transitional) separation bubble (LSB), and is typically associated with flows at low to moderate Reynolds numbers. When present on an airfoil, laminar separation bubbles have a marked effect upon aerodynamic performance. Drag forces are typically increased, and the presence of a separation bubble may determine stall behaviour (Gault 1957). The phenomenon of bubble bursting, where a small increase in incidence leads to a sudden increase in bubble length, causes a dramatic loss in aerodynamic performance and hence is an important consideration in low-Reynolds-number airfoil design. 
Using the results of Gaster (1967), Horton (1969) was the first to describe the time-averaged structure of a laminar separation bubble, and proposed an empirical model for predicting bubble behaviour. Despite refinements such as the use of the $\mathrm{e}^{n}$ transition prediction method, modelling of low-Reynolds-number effects and the dependence on background turbulence levels, present day models do not adequately predict bubble bursting or unsteady behaviour.

More recently, advances in understanding of laminar separation bubbles have been made via numerical methods. The first numerical simulations of separation bubbles were limited either to two-dimensional analysis (Pauley, Moin \& Reynolds 1990), or only studied primary/linear instability and did not resolve transition (Pauley 1994; Rist 1994). Being less computationally expensive, linear stability analysis could be performed before fully resolved direct numerical simulations (DNS) were possible. Hammond \& Redekopp (1998) performed local analysis of separated boundary layer profiles in order to determine whether absolute instability could be observed, as it had been for separated shear layers (Huerre \& Monkewitz 1985) and bluff-body wakes (Hannemann \& Oertel 1989). For certain profiles local absolute instability was observed, depending on both the maximum reverse flow and the height of the reverse flow region. Similar criteria were investigated and confirmed later by Rist \& Maucher (2002). Hammond \& Redekopp (1998) found that for profiles at $R e_{\delta^{*}}=10^{3}$, a minimum reverse flow velocity of $20 \%$ was required to observe local absolute instability. Theofilis (2000) performed global linear stability analysis of a laminar separation bubble, specifying a spanwise wavenumber, $\beta$, and computing the resultant two-dimensional disturbance eigenvectors. A temporally growing stationary mode was observed, associated with unsteadiness at the reattachment point but not affecting the separation point. Growth rates were found to be significantly lower than those associated with amplification of convective instabilities within the shear layer; however Theofilis (2003) suggests that the existence of this mode may potentially be relevant to the phenomenon of vortex shedding from separation bubbles as observed by Pauley et al. (1990).

The first numerical simulations to fully resolve transition to turbulence within a laminar separation bubble were conducted by Alam \& Sandham (2000) and Spalart \& Strelets (2000). Alam \& Sandham (2000) performed DNS of a laminar separation bubble on a flat surface, induced by upper boundary transpiration. Performing linear stability analysis on analytic velocity profiles similar to those observed in the DNS, Alam \& Sandham found that reverse flow greater than $15 \%$ would be required in order to sustain absolute instability, compared to an observed reverse flow of only $4-8 \%$. As a result, it was concluded that the transition process was driven by convective instability. Spalart \& Strelets (2000) conducted DNS of a laminar separation bubble for the purpose of assessing turbulence models. No unsteadiness was introduced and inflow disturbances were less than $0.1 \%$ of the free-stream velocity; however transition to turbulence was still observed. As a result the study stated that entry-region disturbances (referring to Tollmien-Schlichting, or TS, type waves) could be discarded as the mechanism behind transition; however the study also stated that the magnitude of reverse flow present was unlikely to be sufficient to sustain absolute instability. In the study of Spalart \& Strelets three-dimensionality was observed to occur rapidly, with no clear regions of primary or secondary instability, whereas Alam \& Sandham observed $\Lambda$-vortex-induced breakdown. Hence the first two fully resolved DNS of laminar separation bubbles apparently observed different instability mechanisms leading to transition, and different transitional behaviour. 
Marxen et al. (2003) performed a combined DNS and experimental study of an LSB on a flat plate. Periodic two-dimensional disturbances were introduced upstream of separation, and three-dimensionality was introduced via a spanwise array of spacers. The separated shear layer was observed to roll up to form vortices which subsequently broke down to turbulence. The same configuration was studied further by Lang, Rist \& Wagner (2004) and again by Marxen, Rist \& Wagner (2004) in order to quantify the respective roles of two-dimensional and three-dimensional disturbances. Marxen et al. concluded that transition was driven by convective amplification of a two-dimensional TS wave, which also determined the length of the bubble, and that the dominant mechanism behind transition is an absolute secondary instability in a manner first proposed by Maucher, Rist \& Wagner (1997), and investigated further by Maucher, Rist \& Wagner (1998).

It is clear that numerical simulations of separation bubbles can differ markedly in behaviour. Unlike wake and shear layer flows, the presence of regions of absolute instability within laminar separation bubbles has not yet been confirmed by linear stability analysis of DNS or experimental velocity profiles, and stability characteristics of separation bubbles not well defined in all cases. With continued advances in computing power, it is now possible to perform DNS of laminar separation bubbles on full airfoil configurations. This contrasts with previous numerical studies, which have been limited to bubbles on flat plates or other simplified geometries in order to reduce the computational cost. The advantage of studying full airfoil configurations is that the bubble can interact more strongly with the potential flow, in particular via the Kutta condition at the trailing edge. The bubble will be closer in nature to those observed under flight conditions, and the influence of the bubble behaviour on the aerodynamic performance of the airfoil can be observed directly.

The purpose of the current study is to investigate the dependence of bubble behaviour on the presence of boundary layer disturbances, and to investigate the role of instability mechanisms in separation bubble transition. First, data from both forced and unforced three-dimensional simulations of a laminar separation bubble on a NACA-0012 airfoil will be compared. Classical linear stability analysis will then be performed upon the time-averaged flow fields obtained from the DNS, in order to determine whether any local absolute instability is present. Finally, a series of computationally inexpensive simulations will be presented, intended to explain the self-sustained transition to turbulence observed in the first part of the study.

\section{Direct numerical simulations}

\subsection{Simulation geometry}

The chosen airfoil geometry is a NACA-0012, extended to include a sharp trailing edge and rescaled to unit chord length. The coordinate system for the curvilinear $\mathrm{C}$-type grids used in all simulations is given in figure 1. Grids are equidistantly spaced in the $z$-direction for three-dimensional simulations. The two parameters governing domain size are the wake length $W$, and the domain radius $R$. The airfoil chord is used as the reference length scale and the coordinate system is defined such that the trailing edge is located at $(x, y)=(1,0)$.

\subsection{Governing equations}

All simulations were run at a Reynolds number based on airfoil chord of $R e_{c}=5 \times 10^{4}$, and Mach number $M=0.4$ unless otherwise stated. A compressible-flow formulation was chosen so that sound waves originating at the trailing edge could also be studied 


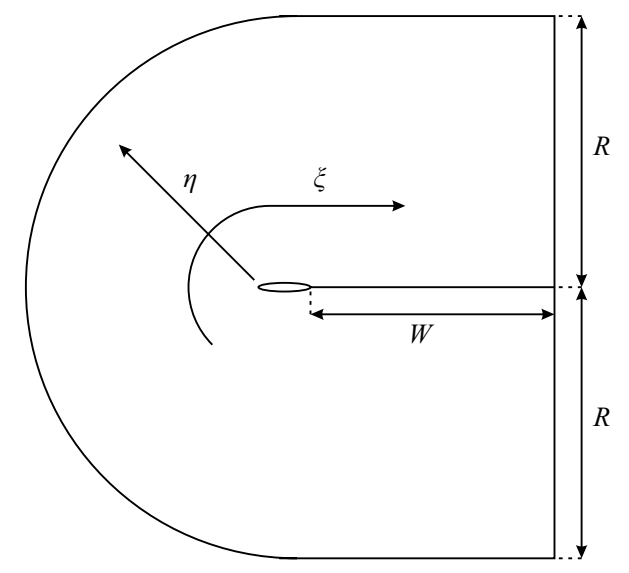

FIGURE 1. Topology of the computational domain.

(Sandberg, Sandham \& Joseph 2007). The DNS code directly solves the unsteady, compressible Navier-Stokes equations, written in curvilinear form as

$$
\frac{\partial \boldsymbol{Q}}{\partial t}+\frac{\partial \boldsymbol{E}}{\partial \xi}+\frac{\partial \boldsymbol{F}}{\partial \eta}+\frac{\partial \boldsymbol{G}}{\partial z}=\frac{\partial \boldsymbol{R}}{\partial \xi}+\frac{\partial \boldsymbol{S}}{\partial \eta}+\frac{\partial \boldsymbol{T}}{\partial z} .
$$

The conservative vector $\boldsymbol{Q}$, inviscid flux vectors $\boldsymbol{E}, \boldsymbol{F}$ and $\boldsymbol{G}$, and the viscous vector terms $\boldsymbol{R}, \boldsymbol{S}$ and $\boldsymbol{T}$ are defined as

$$
\begin{aligned}
& \boldsymbol{Q}=\left(\begin{array}{c}
\rho \\
\rho u \\
\rho v \\
\rho w \\
E_{t}
\end{array}\right), \quad \boldsymbol{E}=\left(\begin{array}{c}
\rho U \\
\rho u U+p \xi_{x} \\
\rho v U+p \xi_{y} \\
\rho w U \\
\left(E_{t}+p\right) U
\end{array}\right) \\
& \boldsymbol{F}=\left(\begin{array}{c}
\rho V \\
\rho u V+p \eta_{x} \\
\rho v V+p \eta_{y} \\
\rho w V \\
\left(E_{t}+p\right) V
\end{array}\right), \quad \boldsymbol{G}=\left(\begin{array}{c}
\rho w \\
\rho u w \\
\rho v w \\
\rho w w+p \\
\left(E_{t}+p\right) w
\end{array}\right) \\
& \boldsymbol{R}=\left(\begin{array}{c}
0 \\
\tau_{x x} \xi_{x}+\tau_{x y} \xi_{y} \\
\tau_{y x} \xi_{x}+\tau_{y y} \xi_{y} \\
\tau_{z x} \xi_{x}+\tau_{z y} \xi_{y} \\
Q_{x} \xi_{x}+Q_{y} \xi_{y}
\end{array}\right), \quad \boldsymbol{S}=\left(\begin{array}{c}
0 \\
\tau_{x x} \eta_{x}+\tau_{x y} \eta_{y} \\
\tau_{y x} \eta_{x}+\tau_{y y} \eta_{y} \\
\tau_{z x} \eta_{x}+\tau_{z y} \eta_{y} \\
Q_{x} \eta_{x}+Q_{y} \eta_{y}
\end{array}\right), \quad \boldsymbol{T}=\left(\begin{array}{c}
0 \\
\tau_{x z} \\
\tau_{y z} \\
\tau_{z z} \\
Q_{z}
\end{array}\right),
\end{aligned}
$$

where $\rho$ is the fluid density, $u, v$ and $w$ are velocity components in the Cartesian $x, y$ and $z$ directions, $p$ is the pressure, and $E_{t}$ is the total energy per unit volume, defined as

where

$$
E_{t}=\rho e+\frac{1}{2} \rho(u u+v v+w w)
$$

$$
e=\frac{T}{\gamma(\gamma-1) M^{2}} .
$$


Metric terms are defined as

$$
\xi_{x}=\frac{y_{\eta}}{J}, \quad \eta_{x}=-\frac{y_{\xi}}{J}, \quad \xi_{y}=-\frac{x_{\eta}}{J}, \quad \eta_{y}=\frac{x_{\xi}}{J},
$$

noting that terms $\xi_{z}$ and $\eta_{z}$ are both equal to zero for computational grids with no spanwise variation, as used in the current study, and the Jacobian $J$ is defined as

$$
J=x_{\xi} y_{\eta}-x_{\eta} y_{\xi} \text {. }
$$

The contravariant velocities $U$ and $V$ are defined as

$$
U=\xi_{x} u+\xi_{y} v, \quad V=\eta_{y} v+\eta_{x} u,
$$

and the stress terms $\tau_{i j}$ as

$$
\tau_{i j}=\frac{\mu}{R e}\left(\frac{\partial u_{i}}{\partial x_{j}}+\frac{\partial u_{j}}{\partial x_{i}}\right)-\frac{2}{3} \frac{\mu}{R e} \frac{\partial u_{k}}{\partial x_{k}} \delta_{i j} .
$$

The terms $Q_{i}$ comprise the conduction and work terms of the energy equation,

$$
Q_{i}=-q_{i}+u_{j} \tau_{i j}
$$

where

$$
q_{i}=\frac{-\mu}{(\gamma-1) M^{2} \operatorname{RePr}} \frac{\partial T}{\partial x_{i}} .
$$

Viscosity is calculated using Sutherland's law,

$$
\mu=T^{3 / 2} \frac{1+C}{T+C}, \quad C=0.368 \dot{6},
$$

and finally, the perfect gas law relates $p, \rho$ and $T$

$$
p=\frac{\rho T}{\gamma M^{2}} .
$$

The primitive variables $\rho, u, v$ and $T$ have been non-dimensionalized by the freestream conditions and dimensionless parameters $\operatorname{Re}, \operatorname{Pr}$ and $M$ are defined using free-stream (reference) flow properties. The ratio of specific heats is specified as $\gamma=1.4$ and the Prandtl number as $P r=0.72$.

\subsection{Numerical method}

Fourth-order-accurate central differences utilizing a five-point stencil are used for spatial discretization. Fourth-order accuracy is extended to the domain boundaries by use of a Carpenter boundary scheme (Carpenter, Nordström \& Gottlieb 1999). No artificial viscosity or filtering is used. Instead, stability is enhanced by entropy splitting of the inviscid flux terms in combination with a Laplacian formulation of the viscous terms (Sandham, Li \& Yee 2002). The explicit fourth-order-accurate Runge-Kutta scheme is used for time-stepping, and all cases were run with a constant time step of $\Delta t=1 \times 10^{-4}$. Appropriate boundary conditions must be applied to avoid unphysical reflections. At the free-stream $\left(\eta^{+}\right)$boundary, where the only disturbances likely to reach the boundary will be in the form of linear waves, an integral characteristic boundary condition is applied (Sandhu \& Sandham 1994). At the downstream exit boundary $\left(\xi^{ \pm}\right)$, which will be subject to the passage of nonlinear fluid structures, a zonal characteristic boundary condition (Sandberg \& Sandham 2006) is applied for increased effectiveness. At the airfoil surface an adiabatic, no-slip condition is applied. 


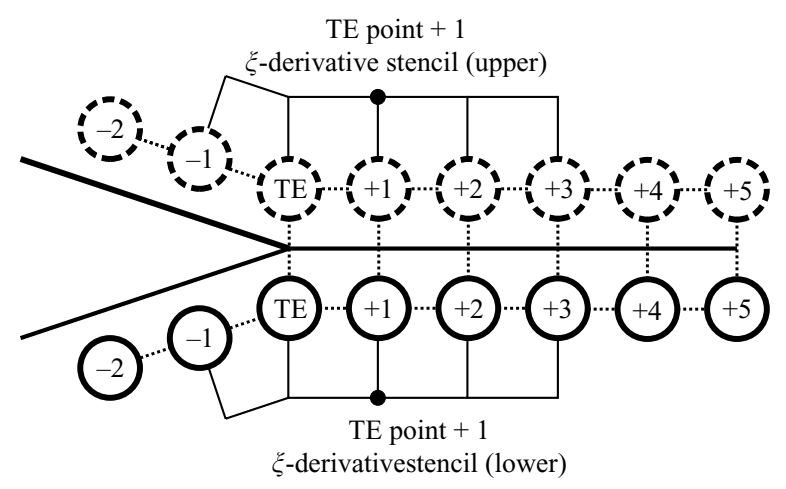

FIGURE 2. $\xi$-derivative stencils on the upper and lower side of the wake cut for the first point downstream of the trailing edge.

Along the wake cut, grid points are effectively duplicated, i.e. points on the line $\eta=0$ for the region above the wake cut are coincident with points on the line $\eta=0$ for the region below the wake cut. This necessitates special treatment for the first two points downstream of the trailing edge, since derivatives computed using the fourthorder central difference scheme will use different stencils depending on whether the derivative is evaluated on the upper or lower airfoil surface. This is illustrated in figure 2 for the first point downstream of the trailing edge. The points on the upper and lower branch cut occupy the same physical space, and to allow the two points to differ in value would be incorrect. In order to solve this problem, when metric terms are computed at the start of the simulation, for the first two points downstream of the trailing edge all terms involving $\xi$-derivatives are averaged across the wake cut. This means that the points on either side of the branch cut will use the same metric terms, thus solving the same equations, and possessing the same values for conservative variables. The trailing-edge point itself is represented by two grid points, one on the upper airfoil surface and one on the lower, which are allowed to differ in value.

The code is based upon an existing code that has been previously validated for compressible turbulent plane channel flow (Sandham et al. 2002), and more recently has been demonstrated to accurately represent the development of hydrodynamic instabilities (Sandberg et al. 2007). The code used in the current study is different in that it is applied to a curvilinear C-type grid with wake connection; however the same metric terms were used in previous versions of the code.

Volume forcing is applied to the $x$ - and $y$-momentum equations in the threedimensional simulation, the goal being to introduce three-dimensional disturbances that are amplified in the free-shear layer and subsequently break down to turbulence. Forcing is applied about the location $(x, y)=(0.1,0.129)$, corresponding to a point within the boundary layer of the time-averaged solution, and is periodic in both time and span. A cosine function is used to smoothly ramp the forcing terms from a maximum at the centre of the forcing location to zero at radius $5 \times 10^{-3}$ from the forcing location. Frequencies were chosen based on linear stability analysis of velocity profiles extracted from the time-averaged two-dimensional flow field, and forcing was applied at several spanwise wavenumbers. The amplitude of velocity disturbances introduced by the forcing is $0.1 \%$ of the free-stream velocity. Details of forcing parameters are given in table 1 , where $\omega=2 \pi f$, with $f$ the frequency, and $\beta$ the spanwise wavenumber. 


$\begin{array}{cc}\omega & \beta \\ 48.76 & 2 \pi / L_{z} \\ 53.60 & 6 \pi / L_{z} \\ 53.60 & 8 \pi / L_{z}\end{array}$

TABLE 1. Forcing parameters.

$\begin{array}{lccc}\text { Grid } & \mathrm{G} 1 & \mathrm{G} 2 & \mathrm{G} 3 \\ R & 5.3 & 5.3 & 7.3 \\ W & 5 & 5 & 5 \\ N_{\xi} & 2001 & 2570 & 2570 \\ N_{\eta} & 440 & 440 & 692 \\ N_{\text {foil }} & 541 & 1066 & 1066 \\ N_{\text {wake }} & 1462 & 1506 & 1506\end{array}$

TABLE 2. Domain and grid dimensions for grid resolution investigation at $R e_{c}=5 \times 10^{4}, \alpha=5^{\circ}$.

\subsection{Grid resolution of the two-dimensional case}

Resolution requirements for direct simulation of the flow around an airfoil at incidence are complex, since a variety of fluid phenomena are present and must be resolved. Grid generation for high-order non-dissipative codes is non-trivial, and is achieved by an iterative approach. The presence of under-resolved flow phenomena results in numerical oscillations, particularly in sensitive quantities such as density gradient or other derivative quantities. By analysing simulation results, locations of poor resolution may be identified by such oscillations. A new grid is then generated, with the purpose of improving the resolution at the necessary locations, and the flow-field data interpolated onto the new grid. The simulation is then run on the new grid and the results analysed in order to assess whether resolution issues have been eliminated. The process is repeated as often as necessary, and avoids the need for starting simulations from scratch. The iterative method of grid production is particularly useful for the case of an airfoil with laminar separation bubble, since $a$ priori grid requirements are not known for all regions.

The iterative grid production process has been performed for the current case in two dimensions. Simulations were run on three grids in total, and sufficient data have been recorded to compare the performance of each grid. The first grid, G1, was generated by estimating resolution requirements based on previous studies at lower Reynolds numbers. Two further grids, denoted G2 and G3, were subsequently produced in order to improve the resolution of the simulation. Details of all grids are given in table 2 .

When run in two dimensions, the upper airfoil boundary layer is observed to separate near the leading edge of the airfoil. The separated shear layer subsequently rolls up to form vortices, and periodic vortex shedding is observed (figure 3, grid G3). The system of laminar separation, shear-layer roll-up and periodic vortex shedding gives rise to a characteristic time-averaged skin friction coefficient, $\bar{c}_{f}$, distribution and causes the lift coefficient, $C_{L}$, to oscillate. These quantities are compared for grids G1-G3 in figure 4.

When run using grid $\mathrm{G} 1, C_{L}$ oscillates in an almost perfectly periodic fashion. The time-averaged $c_{f}$ distribution exhibits separation, secondary separation (the 


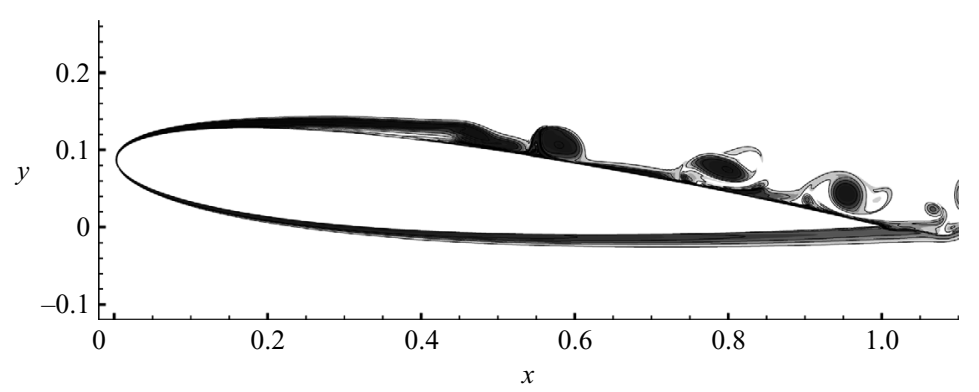

FIGURE 3. Iso-contours of vorticity using 10 levels over the range \pm 150 for grid G3.

(a)

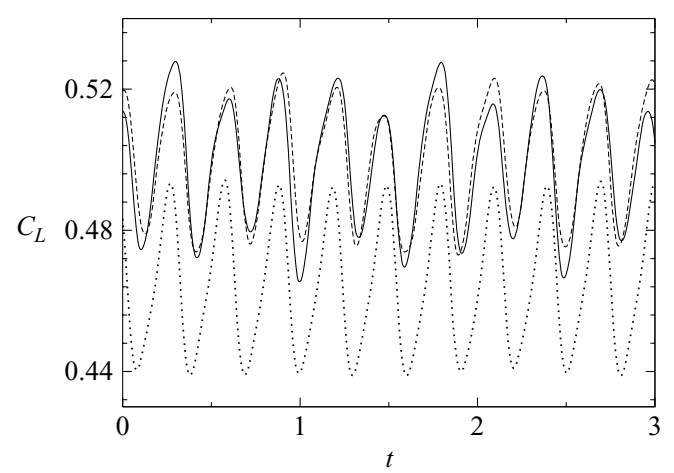

(b)

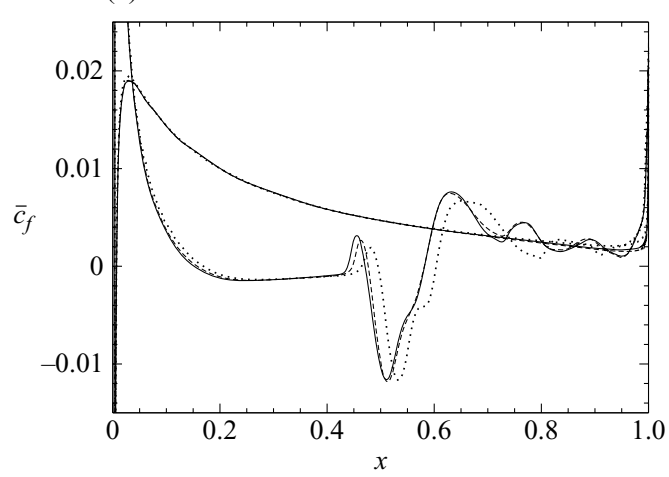

Figure 4. (a) Time-dependent $C_{L}$ and $(b)$ time-averaged $c_{f}$ for grids G1 ( $\left.\cdots\right)$, G2 (--) and G3 (一).

small region of positive $\bar{c}_{f}$ within the larger separated region) and reattachment. Downstream of reattachment a wave-like $\bar{c}_{f}$ distribution is observed. Similar behaviour is observed in two-dimensional simulations performed by Alam \& Sandham (2000), which also exhibited vortex shedding. It appears that this behaviour is caused by the periodic passage of vortices and is unrelated to the presence of a trailing edge. Some evidence of numerical oscillation was observed when iso-contours of vorticity were plotted at sensitive levels, hence grid G2 was generated with an increased streamwise grid resolution over the airfoil surface. When the simulation was continued on grid G2, the mean $C_{L}$ increased, and the time-dependent behaviour became slightly less regular. Numerical oscillations could no longer be observed in hydrodynamic properties. The fundamental frequency of the vortex shedding remained unchanged; however the reattachment point, and hence by inference the vortex shedding location, moved upstream slightly. This alters the wave-like $\bar{c}_{f}$ distribution downstream of reattachment. Grid G3 was generated with increased wall-normal resolution and a larger domain radius compared to grid G2. Minimal difference is observed in $C_{L}$ and $\bar{c}_{f}$ between grids G2 and G3. The behaviour of $\bar{c}_{f}$ in the region of secondary separation changes very slightly; however elsewhere the $\bar{c}_{f}$ distributions are nearly identical. It appears that grid G2 adequately captures the vortex shedding behaviour observed in two dimensions, with no evidence of under-resolution. Grid G3 is more suited to three-dimensional simulations however, since the increased wall-normal resolution is more appropriate for resolving turbulence. 

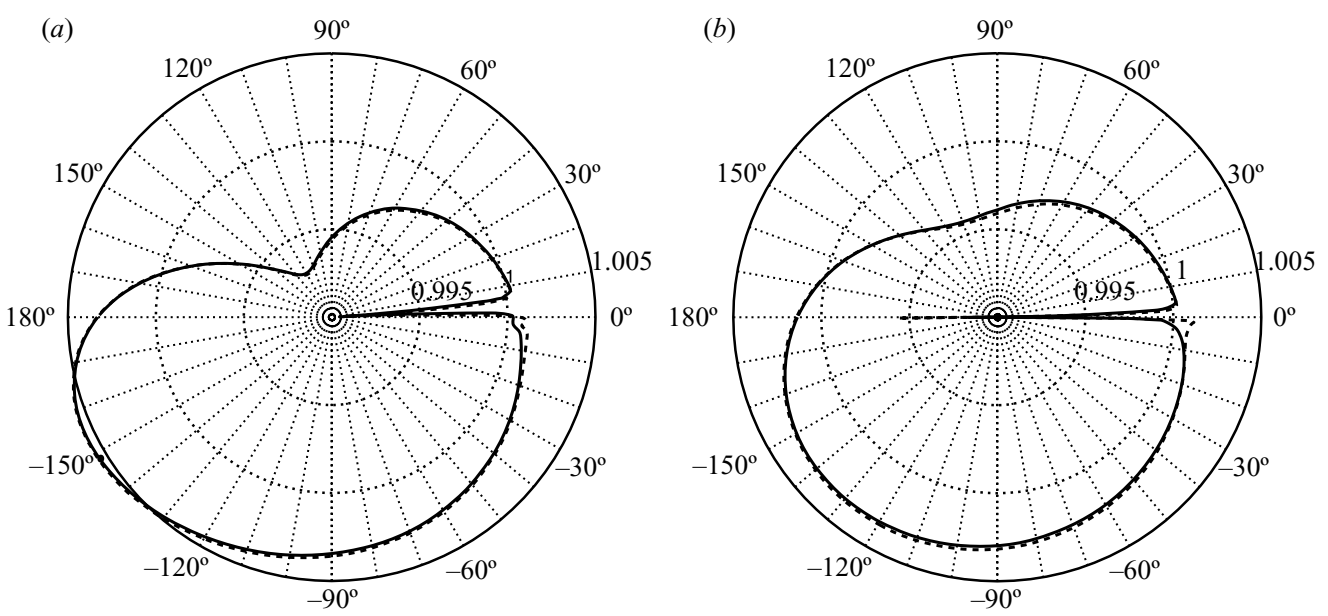

FIGURE 5. Azimuthal variation of $p / p_{\infty}$ over the range 0.99 to 1.005 , at two chords $(a)$ and three chords $(b)$ radius from the airfoil trailing edge, for grids G2 (--) and G3 (-).

\subsection{Domain size}

When choosing the computational domain size, a compromise must be made between capturing the potential flow about the airfoil as fully as possible and minimizing computational expense. The effect of altering domain size may be investigated by considering simulations run using grids G2 and G3 (defined in $\S 2.4$ ); grid G2 is of radius $R=5.3$, whereas grid G3 is of radius $R=7.3$. The azimuthal variation of $p / p_{\infty}$, where $p_{\infty}$ is the free-stream pressure, is plotted in figure 5 for both grids, at a radius of two chords (figure $5 a$ ) and three chords (figure $5 b$ ) from the airfoil trailing edge.

At around $0^{\circ}$ there is a difference of approximately $p / p_{\infty}=1.5 \times 10^{-3}$ between the two grids, presumably caused by differences in resolution in the $\eta$-direction in the wake region; however this is the only significant difference observed. The azimuthal pressure distribution in the potential flow region appears remarkably similar for both cases, and at three chords radius the difference between grids in this region is significantly less than $p / p_{\infty}=1 \times 10^{-3}$. If the radius of the domain were increased beyond seven chords, further changes would be even smaller in amplitude. It appears then that a domain radius of 5.3 airfoil chords adequately captures the potential flow about the airfoil, hence the chosen domain radius of 7.3 chords is more than adequate. The characteristics-based boundary conditions appear sufficiently effective that a comparatively modest domain size is able to capture the potential flow.

\subsection{Extension to three dimensions}

Whilst possible for generating grids for two-dimensional simulations, an iterative grid production method is not suitable for extension to three-dimensional simulations as it would be unfeasibly expensive. Using $\bar{c}_{f}$ predictions from XFoil (Drela \& Giles 1987), the grid resolution over the aft section of the airfoil for grid G3 was compared to turbulent plane channel flow data (Sandham et al. 2002), which suggested that grid G3 would be adequate for the three-dimensional simulation. The spanwise domain width was selected based upon criteria determined from simulations of the flow over a backward-facing step (von Terzi 2004). A domain width of at least 4 times the step height (corresponding approximately to the reattachment length) is necessary to resolve the largest spanwise structures in the case of flow over a backward-facing 


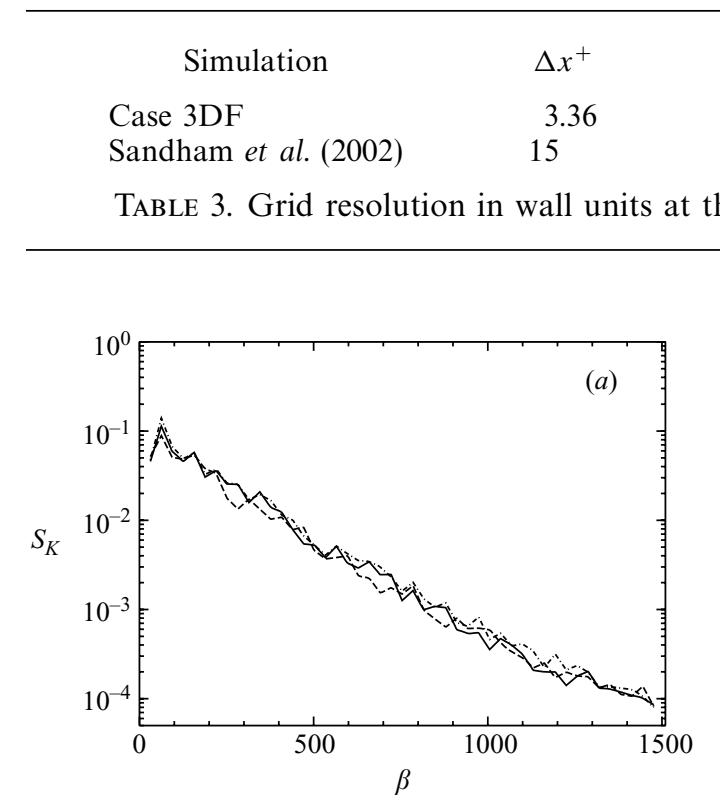

$\begin{array}{lcc}\Delta z^{+} & \Delta y^{+} & N_{\eta}: y^{+}<10 \\ 6.49 & 1.0 & 9 \\ 7.5 & - & 10\end{array}$

the maximum $\bar{c}_{f}$ location for case $3 \mathrm{DF}$.

FIGURE 6. Spanwise power spectra of $K$, integrated over the range $1<y^{+}<50$, taken for case $3 \mathrm{DF}(a)$ and case $3 \mathrm{DU}(b)$ at $x=0.8(-), x=0.9(--)$, and $x=1.0(-\cdot)$.

step. Making an analogy with the maximum bubble height based on displacement thickness, $\delta^{*}$, a domain width $L_{z}=0.2$ was chosen, being 9.6 times the maximum bubble height of $\delta^{*}=2.09 \times 10^{-2}$ and 7.2 times $\delta^{*}$ at the trailing edge. The number of spanwise grid points was chosen to be 96 , again based on the resolution requirements of turbulent plane channel flow and $\bar{c}_{f}$ predictions via XFoil.

During initial stages of three-dimensional simulations, flow-field properties were checked in order to confirm that all fluid structures appeared resolved. A final confirmation of adequate spatial and temporal resolution is provided by a posteriori statistical analysis of the DNS data. Grid resolution in wall units, taken at the maximum turbulent $\bar{c}_{f}$ location observed over all simulations, was found to differ slightly from XFoil predictions, but was still found to be well-resolved based on turbulent plane channel flow criteria. Resolution in wall units for case 3DF (defined in $\S 3$ ), taken at $x=0.612$ where the maximum $\bar{c}_{f}$ of $7.60 \times 10^{-3}$ is observed, is given in table 3.

In order to confirm that turbulent behaviour is resolved over all time and length scales, power spectra of turbulence kinetic energy, defined as $K=\frac{1}{2}\left(\overline{u^{\prime} u^{\prime}}+\overline{v^{\prime} v^{\prime}}+\overline{w^{\prime} w^{\prime}}\right)$, are computed. In order to incorporate a reasonable number of samples, spanwise spectra are integrated over the finite wall-normal distance $1<y^{+}<50$ as well as timeaveraged, using nine data sets taken at intervals of $t=0.7$. Figure 6 displays spanwise power spectra of $K$ taken at three $x$-locations for case 3DF $(a)$ and case 3DU $(b$, defined in $\S 3)$. A roll-off of order $10^{3}$ is observed with increasing wavenumber, comparable with the well-resolved turbulent boundary layer DNS performed by Spalart (1988) using a fully spectral method. Temporal power spectra of $K$ at specific locations are computed by dividing the temporal signal into three segments of equal length, each overlapping by $50 \%$, before computing the spectra of each segment individually and then averaging the spectra over all segments. This improves the quality of spectra, although their frequency range is reduced at the low end $(\mathrm{Hu}$, 

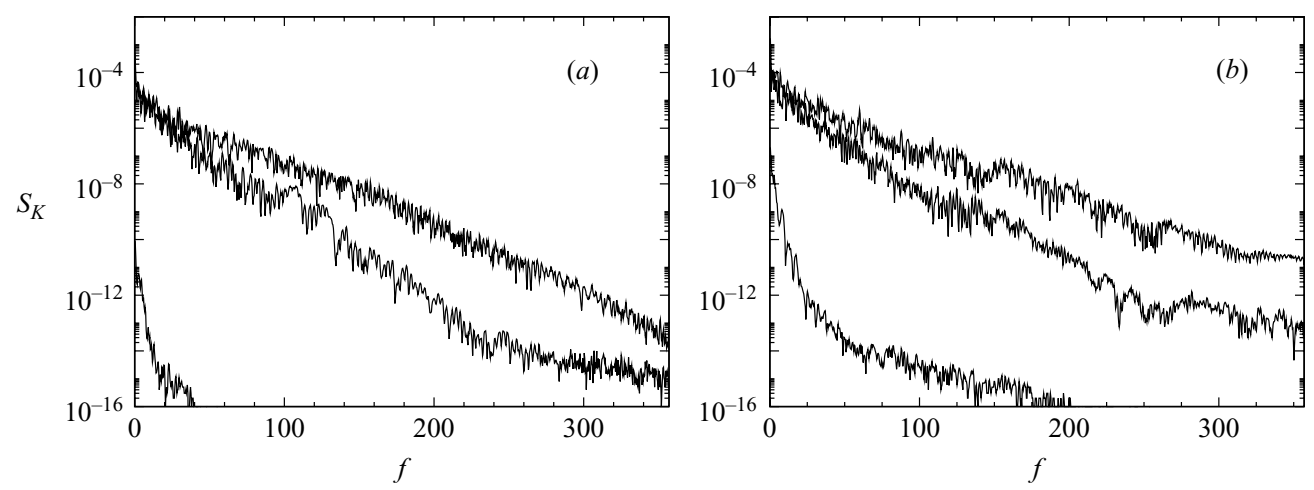

FIGURE 7. Temporal power spectra of $K$, taken at $x=0.9$ at the airfoil mid-span for case 3DF $(a)$, at $y^{+}=12.3,51$ and 313 moving from top to bottom, and for case 3DU $(b)$ taken at $y^{+}=54,13.2$ and 335 moving from top to bottom.

Morfey \& Sandham 2006). Temporal spectra (figure 7) display a minimum roll-off of $10^{6}$ with increasing frequency $f$.

\section{The effect of boundary layer disturbances on separation bubble behaviour}

The first part of the study aims to quantify the effect of boundary layer disturbances on separation bubble behaviour. Results from three DNS will be presented, defined as follows:

Case 2D: A precursory two-dimensional simulation that was run in order to provide a suitable initial condition for the subsequent three-dimensional simulation, and upon which the grid resolution study was performed in section 2.4.

Case 3DF: The flow field from case 2D was extruded in the $z$-direction and threedimensionality was introduced by explicitly adding disturbances via volume forcing. The goal is to excite unstable oblique modes which would subsequently be amplified within the separated shear layer, leading to transition to turbulence.

Case 3DU: After an appropriate amount of statistical data was captured from case $3 \mathrm{DF}$, the simulation was progressed further in time with the forcing removed. The dependence of bubble behaviour on the addition of disturbances could then be investigated.

\subsection{Time-dependent behaviour}

In two dimensions the time-dependent lift coefficient $\left(C_{L}\right)$ exhibits periodic oscillatory behaviour with frequency $f=3.37$ and $\left(C_{L}\right)_{R M S}=0.0172$. As outlined in $\S 2.4$, the cause of this behaviour can be attributed to periodic vortex shedding from the separated shear layer present on the upper airfoil surface (figure 3). This behaviour appears qualitatively similar to that observed by Marxen et al. (2003) and Pauley et al. (1990) in flat-plate simulations, and results in the observed periodic oscillation in lift and drag coefficients, $C_{L}$ and $C_{D}$.

Figure 8 shows a time-history of $C_{L}$ and $C_{D}$ starting at time $t=0$, the start of case 3DF. The time-dependent $C_{L}$ initially displays oscillatory behaviour associated with two-dimensional vortex shedding. This oscillatory behaviour ceases by time $t=2$, whereupon $C_{L}$ increases significantly. At this stage in the flow development, time series of pressure taken within the separated shear layer (figure 9, $x=0.4$ ) clearly exhibit periodic oscillation, associated with the strongly amplified instability waves 

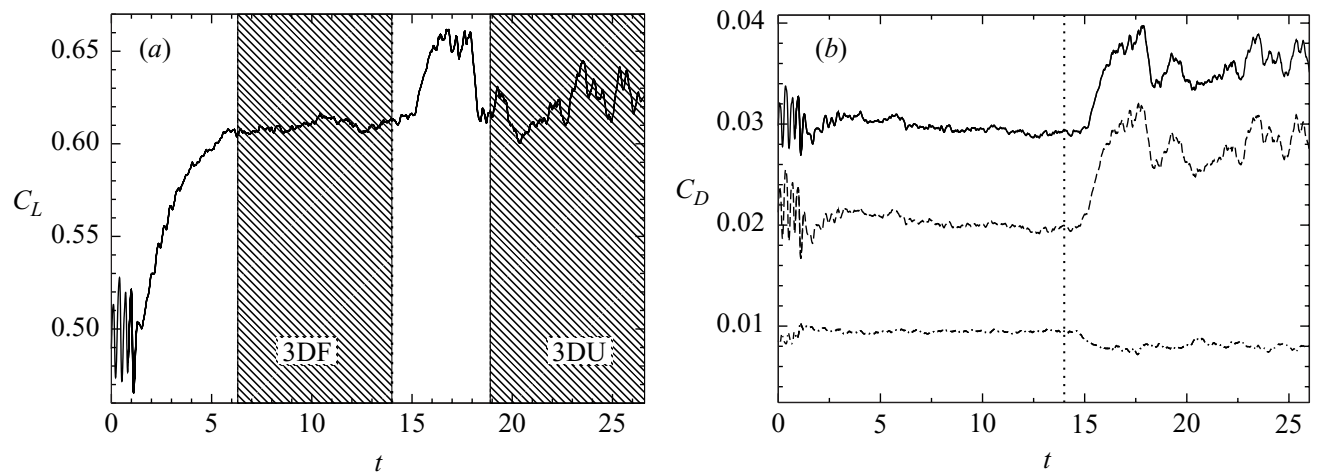

FIGURE 8. (a) Time-dependent lift coefficient, the dotted line indicates the time at which forcing was removed $(t=14)$ and hatched areas indicate periods over which statistical data capture was undertaken. (b) Time-dependent skin-friction drag coefficient (--), pressure drag coefficient $(\cdot-)$, and total drag coefficient $(-)$.

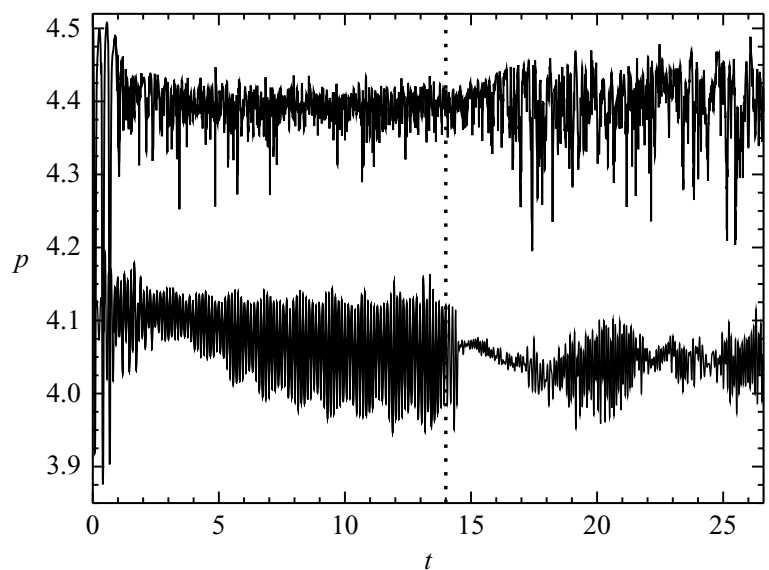

FIGURE 9. Time-dependent pressure within the boundary layer at $x=0.4$ (lower curve) and $x=0.8$ (upper curve). The dotted line indicates the time at which forcing was removed.

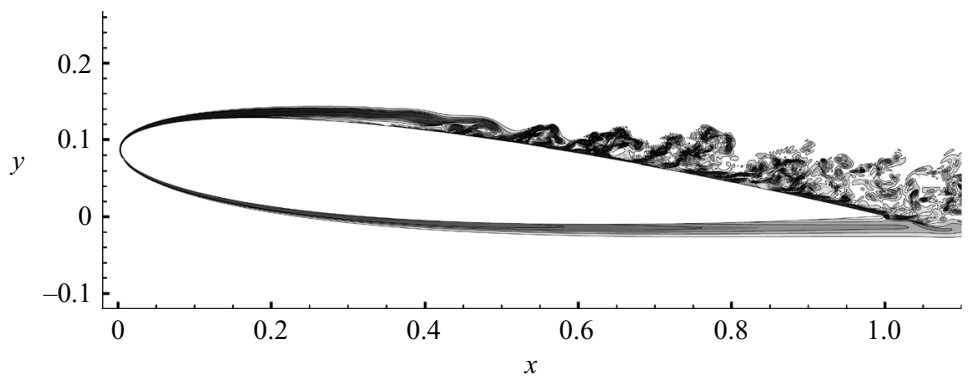

FIGURE 10. Iso-contours of vorticity using 10 levels over the range \pm 150 for case $3 \mathrm{DF} t=14$, taken at mid-span.

induced by the forcing. Downstream of the vortex shedding location, at $x=0.8$, the pressure signal appears to be random, characteristic of turbulent fluctuations passing the measurement location. Instantaneous iso-contours of vorticity taken at the mid-span (figure 10) illustrate that the separated shear layer undergoes transition 

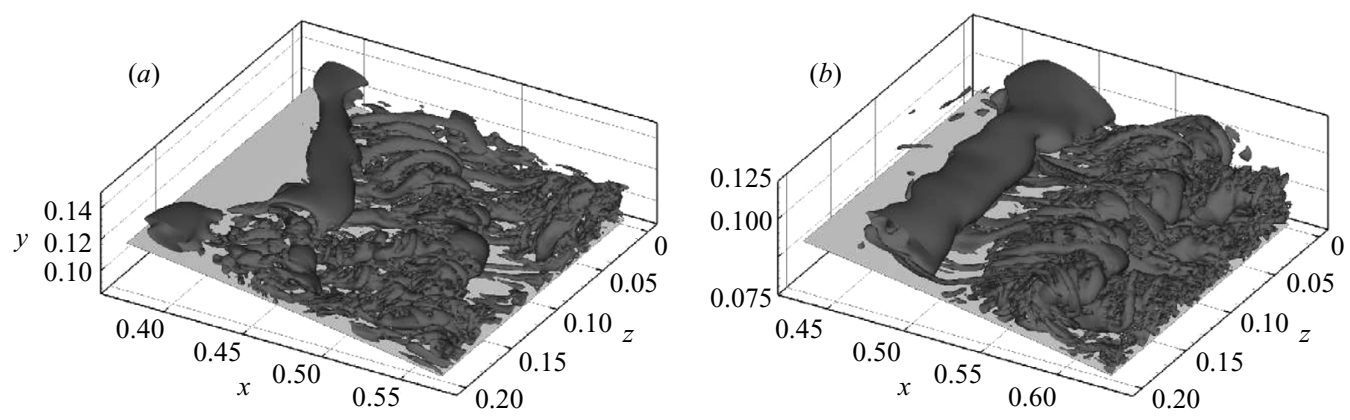

FIGURE 11. Iso-surfaces of the second invariant of the velocity gradient tensor at $Q=500$, for case 3DF at $t=14(a)$ and case 3DU at $t=23.1(b)$.

\begin{tabular}{lcccc}
\hline Case & $C_{L}$ & $C_{D}$ & $C_{D F}$ & $C_{D P}$ \\
2D & 0.499 & 0.0307 & 0.0087 & 0.0220 \\
3DF & 0.615 & 0.0294 & 0.0095 & 0.0199 \\
3DU & 0.621 & 0.0358 & 0.0081 & 0.0278
\end{tabular}

TABLE 4. Time-averaged lift and drag coefficients for all cases.

to turbulence, and that a developing turbulent boundary layer is now present over the aft section of the airfoil. Iso-surfaces of the second invariant of the velocity gradient tensor, $Q$, illustrate structures present in the transition region (figure 11a). Structures within the boundary layer are observed to break down to smaller scales; however no large-scale $\Lambda$-vortices are observed here. After a transient lasting until approximately $t=6.3$, case $3 \mathrm{DF}$ settles to a stationary flow and statistics were taken for $6.3<t<14$. Figure 8 illustrates the data capture period for both three-dimensional simulations.

Case 3DF was then run further in time but with the forcing removed, and the resultant simulation denoted 3DU. Upon removing the forcing, the turbulent behaviour can be monitored by observing pressure fluctuations within the boundary layer (figure 9). It can be seen that downstream of the separation bubble, at $x=0.8$, the pressure fluctuations do not decrease. In fact, the maximum amplitude of pressure fluctuations increases slightly. Oscillations are still observed within the separated shear layer at $x=0.4$; however the signal is lower in amplitude, more intermittent, and no longer dominated by the forcing frequencies as observed in case 3DF. Statistics for case 3DU were taken for $18.9<t<26.6$. At the end of this period of time turbulent fluctuations have still not decreased in amplitude, and the transition to turbulence appears to 'self-sustain'. Iso-surfaces of $Q$ illustrate structures present in the transition region (figure 11b). In contrast to the forced case, much larger structures may be observed, with clear spanwise orientation. These structures persist downstream of the transition region of case $3 \mathrm{DF}$, until they break down into turbulence that still has a strong spanwise coherence. Animations of flow-field properties suggest that the transition process is highly erratic.

\subsection{Statistical analysis}

Time-dependent lift and drag coefficients are given in figure 8 , with the associated time-averaged values in table 4 . It can be seen that whilst removal of forcing leads to a slight increase in $C_{L}$ and a very slight decrease in friction drag $\left(C_{D F}\right)$, pressure 

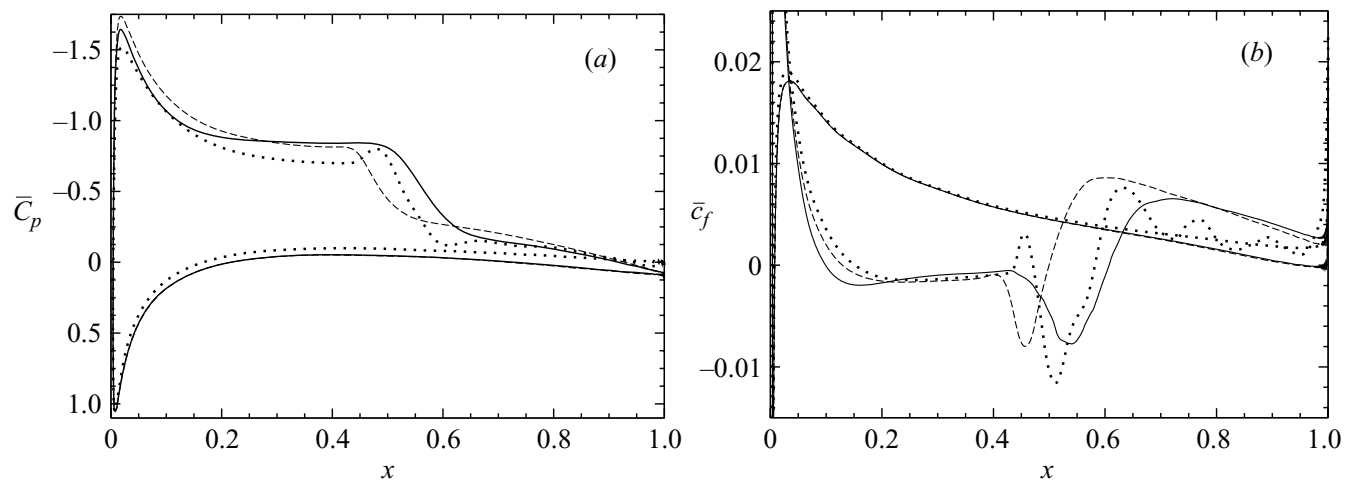

FIgURE 12. Time-averaged distributions of $C_{p}(a)$ and $\bar{c}_{f}(b)$ for case $2 \mathrm{D}(\cdots)$, case 3DF $(--)$ and case $3 \mathrm{DU}(-)$.

$\begin{array}{lcc}\text { Case } & x_{\text {sep }} & x_{\text {reatt }} \\ \text { 2D } & 0.151 & 0.582 \\ \text { 3DF } & 0.128 & 0.500 \\ \text { 3DU } & 0.099 & 0.607\end{array}$

TABLE 5. Time-averaged separation and reattachment points.

drag $\left(C_{D P}\right)$ is subject to a significant increase. The net effect is to decrease $L / D$ from 21.1 to 17.2 , hence it appears that the presence of forcing significantly improves the aerodynamic performance of the airfoil while requiring little energy input. It is conceivable that the introduction of steady disturbances could result in similar performance improvement, whilst requiring zero energy input. For example, Kerho et al. (1993) achieved significant drag reduction for the case of an airfoil with separation bubble by employing vortex generators. An advantage of using timeperiodic forcing however, would be that it could be disabled when not required.

Time-averaged pressure coefficient $\left(C_{p}\right)$ distributions are plotted in figure $12(a)$. In all cases a pronounced pressure plateau is visible on the upper airfoil surface, illustrating the presence of a separation bubble. Comparing cases 3DF and 3DU, it can be seen that the length of the pressure plateau has increased significantly in the unforced case, whereas downstream of the bubble the $C_{p}$ distributions are similar. The slight $C_{L}$ increase observed in case $3 \mathrm{DU}$ is due to the increased length of the pressure plateau. The increase in $C_{D P}$ for case $3 \mathrm{DU}$ can also be attributed to the increase in length of the pressure plateau, since pressure recovery is delayed downstream of the point of maximum airfoil thickness, in conjunction with the reduced suction peak observed near the leading edge.

Time-averaged skin friction coefficient $\left(\bar{c}_{f}\right)$ distributions (figure $12 b$ ) give a quantitative measure of bubble length (table 5). Comparing the two-dimensional simulation to case $3 \mathrm{DF}$ it can be seen that the bubble length has decreased in the forced three-dimensional case. Owing to transition to turbulence and hence increased wall-normal mixing, the reattachment point has moved upstream from $x=0.582$ to $x=0.500$. The separation point has also moved upstream slightly in the forced three-dimensional case. Comparing the three-dimensional cases, it can be seen that removing the forcing has increased the bubble length significantly. The reattachment 

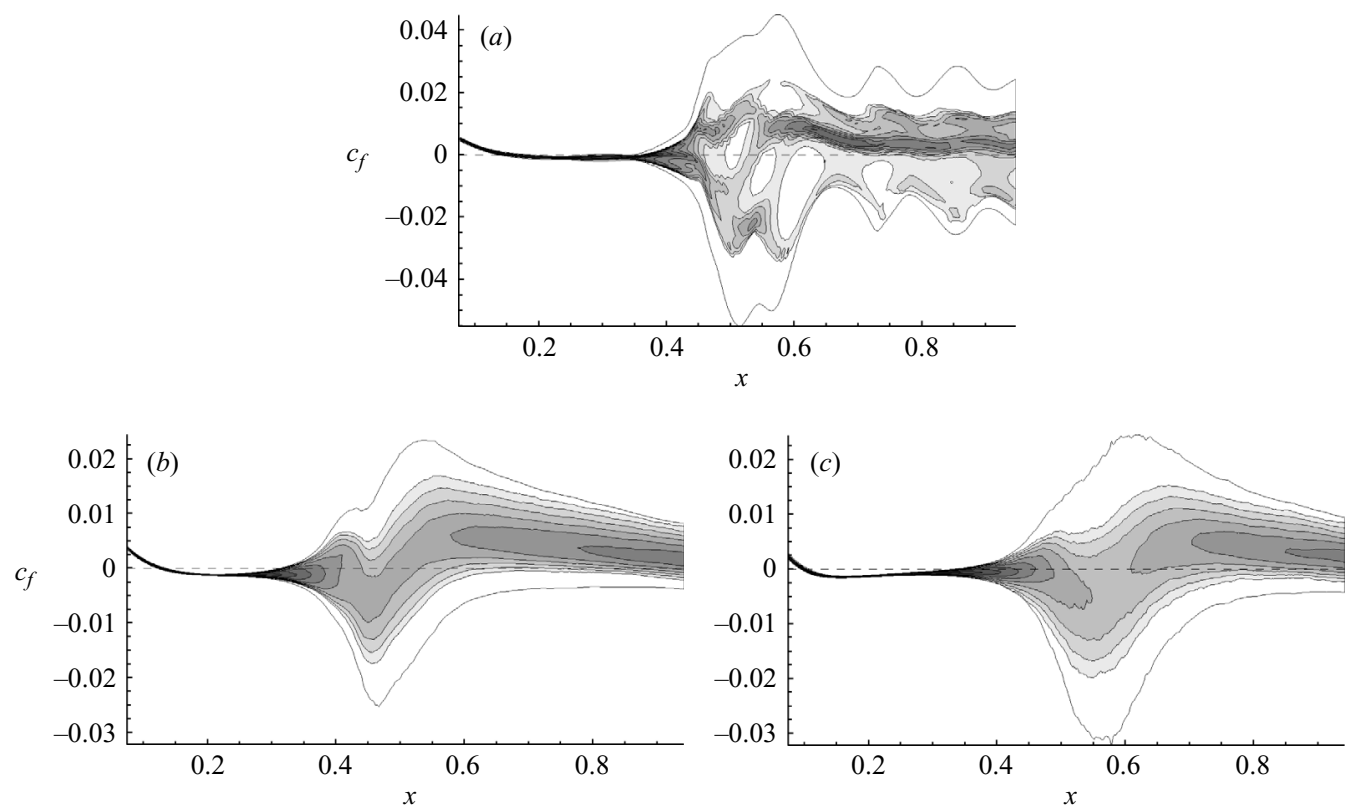

FIGURE 13. Iso-contours of the normalized $c_{f} \mathrm{PDF}, n /\left(S(x) n_{t}\right)$, for case 2D $(a)$, case 3DF $(b)$ and case 3DU $(c)$, using 12 levels exponentially distributed over the range 2 to 1000.

point has moved from $x=0.500$ in case $3 \mathrm{DF}$ to $x=0.607$ in case $3 \mathrm{DU}$. The $\bar{c}_{f}$ peak downstream of transition decreases upon removal of forcing, resulting in the slight decrease in $C_{D F}$ observed in case 3DU.

The time-dependent nature of separation can be investigated by computing probability density functions (PDFs) of $c_{f}$. Ordinarily PDFs are constructed using a fixed number of 'bins' over a constant $c_{f}$ range. In the present study, time-dependent $c_{f}$ behaviour was observed to vary dramatically with $x$-location, making this approach unsatisfactory. Instead, for each $x$-location the PDF was constructed using 30 bins equally spaced over three standard deviations about the mean $c_{f}$. PDF bounds are thus given by the equation

$$
\widetilde{c_{f}}(x, i)=\bar{c}_{f}(x)-3 S(x)+\frac{i-1}{29} 6 S(x), \quad \text { for } i=1,30,
$$

where $S(x)$ is the standard deviation of $c_{f}$ at location $x$. Using different upper and lower bounds for each $x$-location means that the area under the PDF varies with $x$. To avoid this, the normalized PDF (i.e. $N / N_{\text {total }}$, where $N$ is the number of samples in a given bin and $N_{\text {total }}$ is the total number of samples across all bins) is divided by the standard deviation, $S(x)$. This ensures the area under the PDF is constant.

Iso-contours of $c_{f}$ PDFs for a finite $x$-region on the upper airfoil surface are plotted for each case. Figure 13 shows iso-contours of $c_{f}$ PDFs for case 2D (figure 13a), case 3DF (figure 13b) and case 3DU (figure 13c). The upper and lower PDF boundaries represent $c_{f}$ at three standard deviations from the mean; hence where the PDF is very narrow $c_{f}$ varies only little with time, whereas where the PDF is wide $c_{f}$ varies strongly. Upstream of transition $c_{f}$ displays little temporal variation in either case 3DF or case 3DU, confirming that in this region the flow is approximately steady. Similarly, the two-dimensional simulation exhibits little temporal variation upstream of the onset of vortex shedding. Downstream of transition there is considerable 

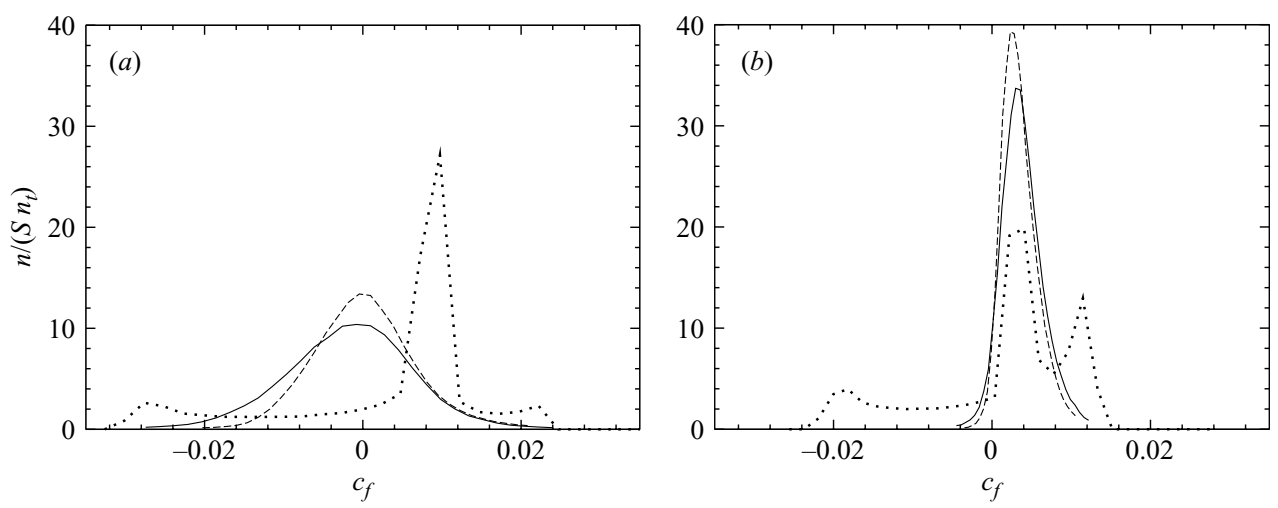

FIGURE 14. Probability density functions of $c_{f}$ taken at reattachment $(a)$, and at $x=0.85(b)$ for case $2 \mathrm{D}(\cdots)$, case $3 \mathrm{DF}(--)$ and case $3 \mathrm{DU}(-)$.

variation in $c_{f}$ for all cases, as illustrated in figure 13 by the comparatively large width of the PDF distributions compared to $\bar{c}_{f}$.

Although all cases exhibit large temporal variation of $c_{f}$ over the aft section of the airfoil, the two-dimensional and three-dimensional PDF distributions appear fundamentally different in this region. The three-dimensional PDF distributions appear smooth and symmetric about the mean $c_{f}$, whereas the two-dimensional PDF exhibits greater temporal variation overall, and in certain locations has more than one maximum. For all cases, downstream of transition (or onset of vortex shedding in the two-dimensional case) there is no location where $c_{f}$ is positive for $100 \%$ of the time, or negative for $100 \%$ of the time. That is to say, even where the time-averaged $c_{f}$ suggests the boundary layer is attached, there will be some degree of reverse flow observed in the instantaneous flow field, and vice versa for separated boundary layer regions. Plotting PDFs at the mean reattachment point (figure 14a), i.e. where $\bar{c}_{f}=0$, illustrates the different distributions of the $c_{f}$ fluctuations. For both three-dimensional cases the time-dependent $c_{f}$ varies strongly over the range \pm 0.02 at this location, and for case $2 \mathrm{D}$ the variation is even greater. To put this into context, the maximum time-averaged $c_{f}$ observed in the attached turbulent boundary layer across all cases was $7.6 \times 10^{-3}$ (figure 12). Hence at the reattachment point, where the time-averaged $c_{f}$ is zero, the instantaneous $c_{f}$ reaches more than double the maximum $\bar{c}_{f}$ observed after reattachment.

Downstream of transition (e.g. figure 14b) the shape of the PDF distribution appears similar for both case $3 \mathrm{DF}$ and case $3 \mathrm{DU}$ : a symmetric distribution about the mean $c_{f}$. The PDF distribution for case 2D is markedly different at this location however, consisting of a skewed distribution exhibiting two peaks at positive $c_{f}$, and a plateau extending to another peak at $c_{f}=-0.02$. For the three-dimensional cases the $c_{f}$ PDF downstream of transition can be approximated by two parameters, $\bar{c}_{f}(x)$ and $S(x)$, with minimal loss of information. The two-dimensional simulation appears to exhibit fundamentally different behaviour however, and cannot be modelled in this fashion.

Spanwise coherence can be determined by computing two-point spanwise correlations of surface pressure, defined as

$$
C_{z_{1} z_{2}}=\frac{S_{z_{1} z_{2}}}{\sigma_{z_{1}} \sigma_{z_{2}}}
$$



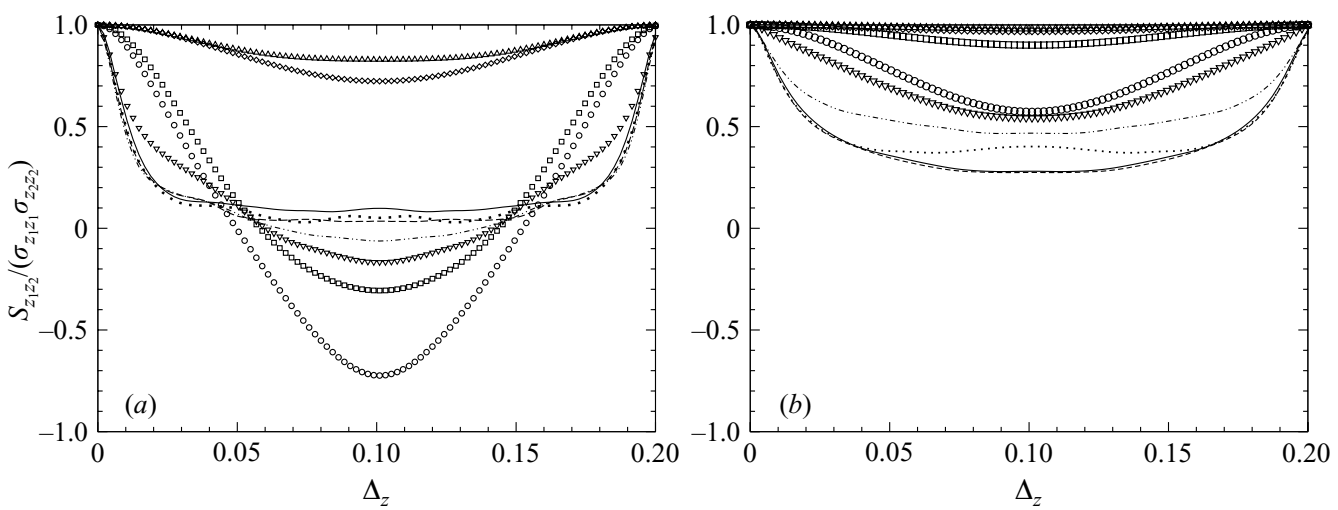

FIGURE 15. Two-point spanwise correlations for case 3DF $(a)$ and case 3DU $(b)$, at $x$-locations $0.1(\diamond), 0.2(\triangle), 0.3(\square), 0.4(\circ), 0.5(\nabla), 0.6(-\cdots), 0.7(\cdots), 0.8(--)$, and $0.9(-)$.

where $S_{z_{1} z_{2}}$ is the covariance of surface pressure for $z_{1}$ and $z_{2}$, and $\sigma_{z_{n}}$ is the standard deviation of surface pressure at $z_{n}$. Two-point spanwise correlations of surface pressure taken at several $x$-locations for case $3 \mathrm{DF}$ are displayed in figure $15(a)$. For $x \leqslant 0.2, C_{z_{1} z_{2}}$ is close to 1 across the entire span, suggesting that the small-amplitude boundary layer fluctuations in this region are primarily two-dimensional. By $x=0.4$ there is strong negative correlation, with $C_{z_{1} z_{2}} \approx-0.75$. For case $3 \mathrm{DF}$ the spanwise wavenumber $\beta=2 \pi / L_{z}$ was forced at double the amplitude of higher wavenumber modes. The strong negative correlation observed at $x=0.4$ can be attributed to the strong amplification within the separated shear layer of perturbations with wavenumber $\beta=2 \pi / L_{z}$ introduced by the volume forcing. Downstream of $x=0.4$, $C_{z_{1} z_{2}}$ decreases in amplitude until by $x=0.7$ there is minimal correlation. This would appear to confirm that the spanwise domain width of $z=0.2$ is sufficiently large.

Case 3DU (figure 15b) exhibits very different behaviour. For $x<0.3$ the surface pressure appears perfectly correlated. In the region $0.3 \leqslant x \leqslant 0.5$ surface pressure becomes slightly less correlated; however unlike case $3 \mathrm{DF}$ no negative correlation is observed. This appears to confirm that the negative correlation observed in case $3 \mathrm{DF}$ is caused by forcing the boundary layer. Downstream of transition surface pressure becomes less correlated, but $C_{z_{1} z_{2}}$ only decreases to around 0.3 . Thus case $3 \mathrm{DF}$ exhibits significant spanwise correlation in surface pressure downstream of transition. However, referring to instantaneous plots of $Q$ (figure 11), in case 3DU large structures are observed with strong spanwise coherence that are not observed in case $3 \mathrm{DF}$. The non-zero correlation observed downstream of transition for case 3DU serves to confirm quantitatively that the turbulence downstream of reattachment retains significant spanwise coherence all the way to the trailing edge.

Iso-contours of turbulent kinetic energy, $K$, (figure 16) show a significant increase in peak $K$ upon removal of forcing (increasing from 0.074 to 0.124 ), thus it appears that the transition process in the unforced case is more energetic than in the forced case. In case 3DU the peak $K$ occurs upstream of the time-averaged reattachment point, whereas in case $3 \mathrm{DF}$ the peak $K$ occurs in the direct vicinity of reattachment, which may explain why the peak $\bar{c}_{f}$ is lower in case 3DU (figure 12).

It is important to note that upon removal of forcing, although the bubble properties change significantly, the bubble does not revert to two-dimensional behaviour. If the bubble were purely convectively unstable, one would expect turbulent fluctuations to convect downstream and ultimately leave the flow over the airfoil in an unperturbed 
(a)

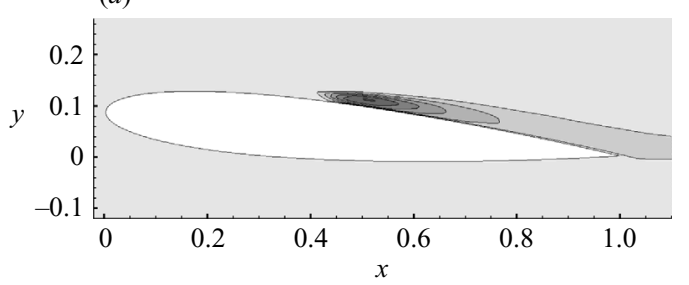

(b)

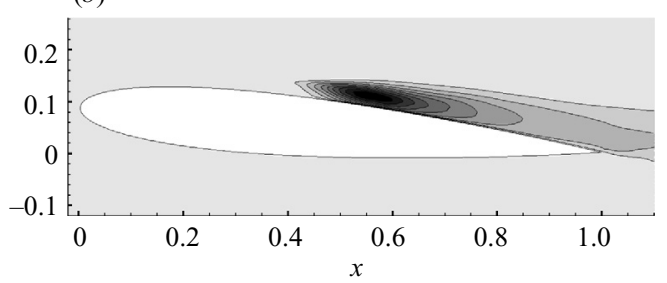

Figure 16. Iso-contours of $K$ for case 3DF $(a)$ and 3DU (b), using 20 levels over the range 0 to 0.11 .

state. This is clearly not the case, and some other local or global instability mechanism must be present in order for the turbulence to self-sustain.

\section{Linear stability analysis}

The presence of self-sustaining turbulence in simulation 3DU raises the question of whether any form of absolute instability is present. In order to determine whether or not this is the case, linear stability analysis of velocity profiles extracted from the timeand span-averaged flow fields of case 3DF, 3DU and the two-dimensional simulation are presented. An incompressible Orr-Sommerfeld solver is used in conjunction with the cusp-map method in order to determine the presence of absolute instability.

\subsection{Governing equations}

Assuming incompressible flow, boundary layer disturbances are assumed to take the form of two-dimensional travelling waves such that

$$
u_{i}^{\prime}=\hat{u_{i}}(y) \mathrm{e}^{\mathrm{i}(\alpha x-\omega t)} \text {. }
$$

The variable $\alpha$ is the complex wavenumber (defined as $\alpha=2 \pi / \lambda$, where $\lambda$ is the disturbance wavelength) and $\omega$ is the complex frequency of the travelling wave (defined as $\omega=2 \pi f$, where $f$ is the disturbance frequency). Wall-normal variation is accounted for in the function $\hat{u}_{\mathrm{i}}(y)$, and the phase velocity is given by $c_{p h}=\omega / \alpha$. The amplitude of instability waves varies as

$$
\mathrm{e}^{\omega_{\mathrm{i}} t-\alpha_{\mathrm{i}} x}
$$

found by expanding (4.1), hence the imaginary part of the wavenumber $\left(-\alpha_{\mathrm{i}}\right)$ corresponds to the spatial growth rate and the imaginary part of the frequency $\left(\omega_{\mathrm{i}}\right)$ corresponds to the temporal growth rate.

Assuming a parallel base flow, for which $\bar{u}=f(y), \bar{v}=0, \mathrm{~d} \bar{u} / \mathrm{d} x=0$, the response of the boundary layer to small-amplitude perturbations of the form given by equation (4.1) may then be predicted by solving the Orr-Sommerfeld equation,

$$
\left(\bar{u}-c_{p h}\right)\left(\frac{\mathrm{d}^{2} \hat{\boldsymbol{v}}}{\mathrm{d} y^{2}}-\alpha^{2} \hat{\boldsymbol{v}}\right)-\frac{\mathrm{d}^{2} \bar{u}}{\mathrm{~d} y^{2}} \hat{\boldsymbol{v}}=-\frac{\mathrm{i} \nu}{\alpha}\left(\alpha^{4} \hat{\boldsymbol{v}}-2 \alpha^{2} \frac{\mathrm{d}^{2} \hat{\boldsymbol{v}}}{\mathrm{d} y^{2}}+\frac{\mathrm{d}^{4} \hat{\boldsymbol{v}}}{\mathrm{d} y^{4}}\right),
$$

for which a full derivation is given in Drazin \& Reed (1981). To solve the OrrSommerfeld equation, a velocity profile $\bar{u}=f(y)$ is specified (extracted from timeaveraged DNS data), hence $\bar{u}(y)$ and $\mathrm{d}^{2} \bar{u} / \mathrm{d} y^{2}$ are known. The Orr-Sommerfeld equation then represents an eigenvalue problem in matrix form $[A] \hat{\boldsymbol{v}}=[B] \hat{\boldsymbol{v}}$, with $\hat{\boldsymbol{v}}$ as the eigenvector, which yields non-trivial solutions for only certain values of $\alpha$ and $c_{p h}$. The eigenvalue problem may be solved in two ways: either by specifying $\alpha$ 
and solving for $\omega$, denoted temporal analysis, or by specifying $\omega$ and solving for $\alpha$, denoted spatial analysis.

\subsection{Numerical method}

The code used for the current study solves the temporal eigenvalue problem, returning $\omega$ for the most unstable eigenmode present. Derivatives are computed using sixth order compact difference stencils (Lele 1992). Sufficient resolution for the derivative scheme is indicated by smooth derivatives of velocity profiles, up to and including the fourth derivative.

In the present study velocity profiles, $\bar{u}(y)$, are extracted from time-averaged DNS data. Grid requirements for DNS are typically different to those of LST analysis; therefore in order to avoid unnecessary computational cost, data from the DNS are interpolated onto a new grid using cubic splines. Grids used for LST analysis are described by the equation

$$
y_{j}=y_{j-1}+a(1+s)^{j-2},
$$

for $2<j<N$, where $N$ is the total number of grid points, and

$$
y_{1}=0 \text {. }
$$

The constant $s$ is the fractional increase in adjacent cell size, and $a$ determines the cell size at $y=0$. In all cases the number of grid points was specified as $N=200$, in conjunction with values $s=0.055$ and $a=1.30 \times 10^{-4}$. Grids used for the stability analysis extend further into the free stream than the grid used for DNS, hence at the domain boundary the velocity profile was smoothly ramped to free-stream conditions. The most unstable eigenmode for a Blasius boundary layer profile at $R e_{\delta^{*}}=1500$, $\alpha=0.2$ was determined, and the resultant value for $\alpha$ was found to agree with the results of Gaster (1978) to the 5th digit for the real part and the 6th digit for the imaginary part.

\subsection{Cusp-map method for determining the presence of absolute instability}

A simple criterion for the presence of absolute instability is the existence of an instability wave possessing zero group velocity, $c_{g}=0$, and a positive temporal growth rate, $\omega_{\mathrm{i}}>0$. The cusp-map technique is a method of looking for the presence of absolute instability based on this criterion, and is the temporal equivalent of Briggs method. A full description of both Briggs method and the cusp-map method is found in Schmid \& Henningson (2001); a brief description necessary to interpret the results follows. Given that a dispersion relation $D$, in this case the Orr-Sommerfeld equation, relates $\alpha$ and $\omega$,

$$
D(\alpha, \omega)=0,
$$

points in the complex- $\omega$-plane will map to points in the complex- $\alpha$-plane and vice versa. The presence of a saddle point in the complex- $\alpha$-plane represents a point where $c_{g}=0$, since for a saddle point

$$
\mathrm{D}(\alpha, \omega)=0, \quad \frac{\partial \mathrm{D}(\alpha, \omega)}{\partial \alpha}=0
$$

and

$$
c_{g}=\frac{\partial \omega}{\partial \alpha}=\frac{\partial \mathrm{D}}{\partial \alpha} / \frac{\partial \mathrm{D}}{\partial \omega}=0 .
$$

Where a saddle point occurs in the complex- $\alpha$-plane, a branch point will occur in the complex- $\omega$-plane. Essentially, the cusp-map method is a systematic procedure 

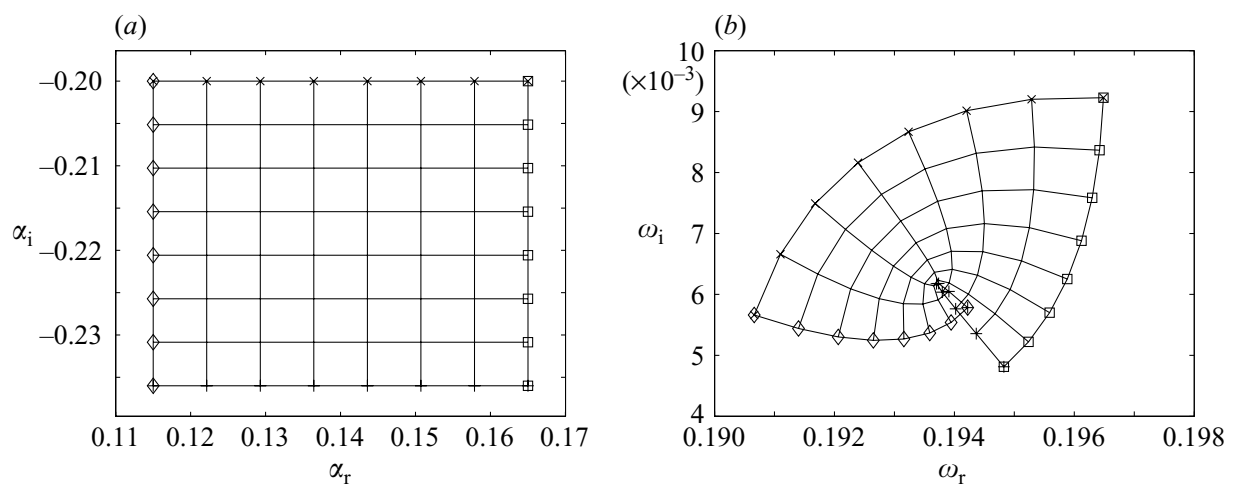

FIGURE 17. Equidistant grid in the complex- $\alpha$-plane $(a)$ and its corresponding map into the complex- $\omega$-plane $(b)$, revealing a cusp associated with $c_{g}=0$ and $\omega_{\mathrm{i}}>0$, as determined for the shear layer profile given by equation (4.9) with $R=1.35$.

for locating saddle and branch point pairs, and hence instability waves with $c_{g}=0$. Lines of constant $\alpha_{\mathrm{r}}$ are plotted in the complex- $\alpha$-plane, and then mapped via the dispersion relation to the complex- $\omega$-plane. A branch point in the complex- $\omega$-plane may be readily observed as a 'cusp' where contours in the complex- $\omega$-plane first cross (figure 17). The presence of a branch point represents an instability wave with $c_{g}=0$. If the branch point is in the lower half of the complex- $\omega$-plane (i.e. $\omega_{\mathrm{i}}<0$ ), the stationary wave is absolutely stable. If the branch point is in the upper half of the complex- $\omega$-plane (i.e. $\omega_{\mathrm{i}}>0$ ), the stationary wave is absolutely unstable.

The method is employed in the current study as follows. First, for the profile of interest, the Orr-Sommerfeld equation is solved for a range of real $\alpha$, in order to determine the envelope of $\alpha_{\mathrm{r}}$ yielding unstable values of $\omega$. The upper and lower limits of this envelope are denoted $\alpha_{\mathrm{r} 1}$ and $\alpha_{\mathrm{r} 2}$. A second sweep is then performed over a range of both $\alpha_{\mathrm{r}}$ and $\alpha_{\mathrm{i}}$, forming an equidistant grid in the complex- $\alpha$-plane (figure 17a). The upper and lower $\alpha_{\mathrm{r}}$ values are chosen as $\alpha_{\mathrm{r} 1}$ and $\alpha_{\mathrm{r} 2}$. The upper and lower limits of $\alpha_{\mathrm{i}}$ are chosen intuitively, for the first attempt, and then refined. The associated map in the complex- $\omega$-plane will either contain a cusp (figure 17b), or else the process can be repeated making adjustments to $\alpha$ in order to locate a cusp. Once a cusp is found, it may be tracked as the boundary layer profile slowly varies, and the corresponding $\alpha$ and $\omega$ noted.

Use of the Orr-Sommerfeld solver in conjunction with the cusp-map method to determine the presence of absolute instability has been validated for analytic wake profiles given in Huerre \& Monkewitz (1985), described by the equation

$$
u(y)=1+R \tanh \left(\frac{y}{2}\right) .
$$

The variation of $\omega_{\mathrm{i}}$ with $R$, determined using the Orr-Sommerfeld solver in conjunction with the cusp-map method and setting the Reynolds number to $R_{\theta}=10^{6}$, is plotted in figure 18 in the vicinity of $\omega_{\mathrm{i}}=0$. Transition from convective to absolute instability was found to occur at $R=1.3156$, compared to $R=1.315$ as reported by Huerre \& Monkewitz using an inviscid approach. For the profile $R=1.315$, values $\omega_{\mathrm{i}}=-1.266 \times 10^{-4}$ and $\omega_{\mathrm{r}}=1.921 \times 10^{-1}$ were determined, compared to $\omega_{\mathrm{i}}=0$ and $\omega_{\mathrm{r}}=1.92 \times 10^{-1}$ as reported by Huerre \& Monkewitz. 


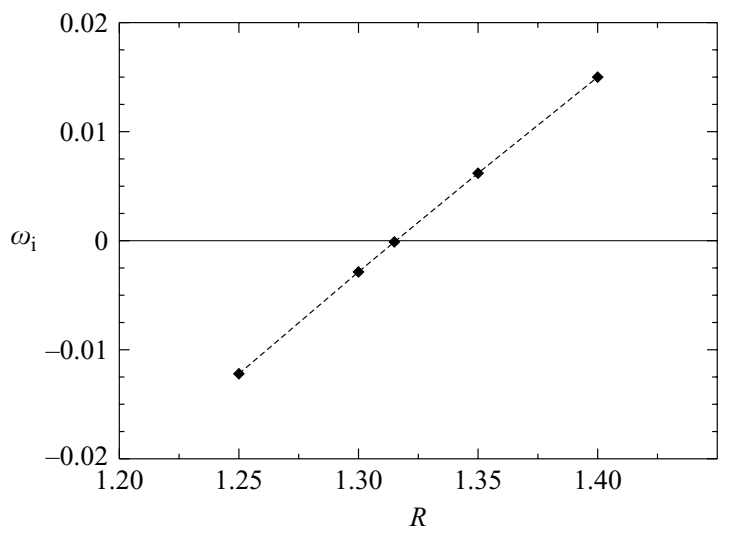

FIGURE 18. Variation with $R$ of complex $\omega$ associated with $c_{g}=0$, for profiles given in Huerre \& Monkewitz (1985).

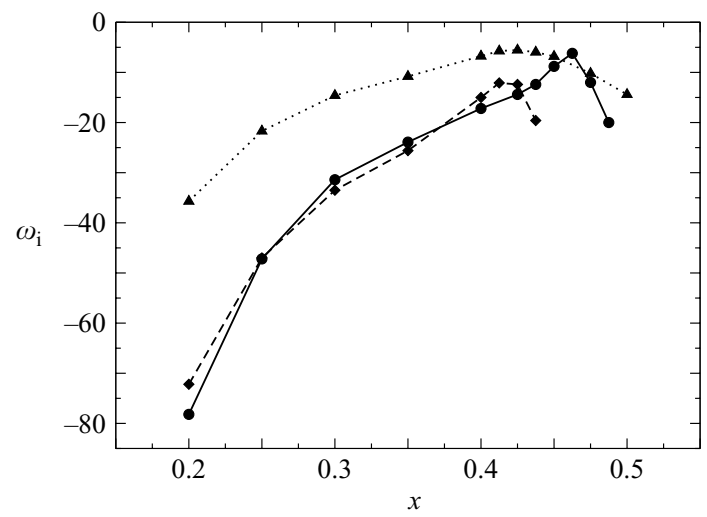

Figure 19. Variation with $x$ of $\omega_{\mathrm{i}}$ associated with $c_{g}=0$, for case 2D $(\bullet-\bullet)$, case 3DF

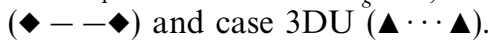

\subsection{Results}

The time- and span-averaged flow fields of cases $3 \mathrm{DF}$ and $3 \mathrm{DU}$, as well as the time-average of the two-dimensional simulation, have been analysed using the OrrSommerfeld solver in conjunction with the cusp-map method. The complex- $\alpha$-plane was swept with a resolution of $\Delta \alpha_{\mathrm{r}}=\Delta \alpha_{\mathrm{i}}=1$. The corresponding resolution in the complex- $\omega$-plane is much higher in the vicinity of a branch point, since $\partial \omega / \partial \alpha \approx 0$. Branch-point singularities associated with zero-group-velocity instability waves have been tracked, traversing the upper airfoil surface from $x=0.1$ until it is no longer possible to locate any branch point within the complex- $\omega$-plane. Imaginary parts of the complex frequency associated with $c_{g}=0$ are plotted in figure 19. In all cases, as the $c_{g}=0$ instability wave is tracked downstream, $\omega_{\mathrm{i}}$ increases with $x$, until a maximum value is reached toward the rear of the separation bubble. After reaching this maximum value, $\omega_{\mathrm{i}}$ decays with further increase in $x$. For all cases, at all locations analysed, $\omega_{i}$ associated with the singularity is negative, hence there is no evidence that absolute instability is present.

For each simulation, a branch point could not be located downstream of a certain $x$-location, unique to that case. Downstream of this location, the Orr-Sommerfeld 
(a)

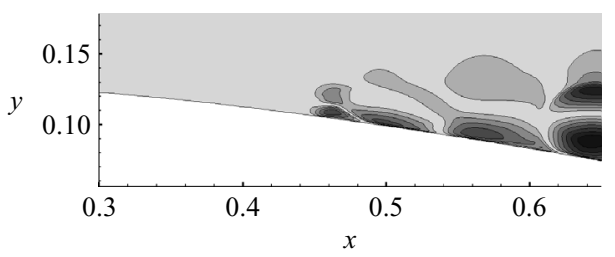

(b)

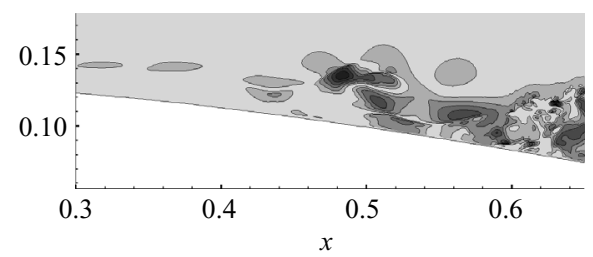

FIGURE 20. Iso-contours of $u^{\prime}$ for case 2D, taken at an arbitrary time within the vortex shedding cycle $(a)$, and for case 3DU taken at time $t=26.6(b)$, using 10 levels over the range $0-1$.

solver returned trivial solutions for regions of the complex- $\alpha$ sweep. Contours of $u^{\prime}$, defined as $u^{\prime}=u-\bar{u}$, illustrate that for all cases the flow field is already highly unsteady at the $x$-position at which the solver fails (figure 20). Depending on the case, $u^{\prime}$ and $v^{\prime}$ lie in the range $0.3-0.7$ at the point where the solver fails. Clearly the assumption of small-amplitude linear perturbations on a steady base flow would anyway be violated under these conditions. The location of maximum reverse flow could therefore not be analysed for any of the simulations. The magnitude of reverse flow observed in each simulation does however allow qualitative discussion.

When normalized by the local boundary layer edge velocity, case 3DF exhibits a maximum reverse flow magnitude of $12.3 \%$. This is less than the critical value of $17 \%$ determined necessary to sustain absolute instability by Alam \& Sandham (2000), for the associated $R e_{\delta^{*}}$ of 1050. Case 3DU exhibits an increased maximum reverse flow of $15.2 \%$. This is only slightly less than the critical value of $16.5 \%$ for $R e_{\delta^{*}}=1350$, determined by Alam \& Sandham. However, Hammond \& Redekopp (1998) determined a higher critical value of $20 \%$ for Falkner-Skan-type boundary layers and Rist \& Maucher (2002) determined that, even in the case of $20 \%$ reverse flow, the wall-normal distance and intensity of the shear layer had to exceed a certain threshold before absolute instability could be observed. The fact that $\mathrm{d} \omega_{\mathrm{i}} / \mathrm{d} x<0$ at the point where the cusp-map method fails suggests that absolute instability would be unlikely to be observed by linear stability analysis if the cusp-map could be continued further downstream. The two-dimensional simulation exhibited the largest magnitude reverse flow of all cases, $22.2 \%$. This is certainly above threshold values observed by Alam \& Sandham (2000) and Hammond \& Redekopp (1998); however, as for case 3DU, the results of Rist \& Maucher (2002) suggest that caution should be exercised before labelling the flow as absolutely unstable. On the one hand, it could be conjectured that the vortex shedding observed is the result of absolute instability; however it may be more useful to consider the shedding to be caused by a global instability mode (Theofilis 2003), resulting in highly unsteady flow for which linear stability analysis is not valid. It is interesting to note, however, that in all cases the maximum $\omega_{\mathrm{i}}$ observed occurs upstream of the location of maximum reverse flow, and appears to decrease with further increase in $x$.

\section{A mechanism for self-sustaining turbulence}

No evidence of absolute instability was observed from the linear stability analysis performed in $\S 4.4$. However, the persistence of turbulence upon removal of forcing suggests that some mechanism other than convective disturbance growth is present. A series of computationally inexpensive simulations was therefore conducted, in order 
(a)

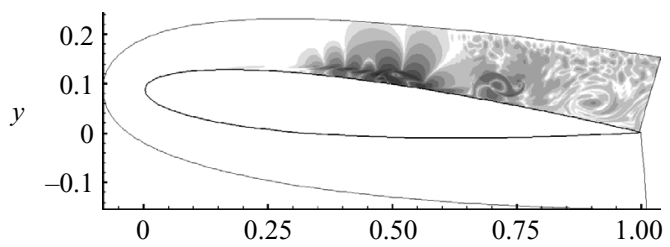

(c)

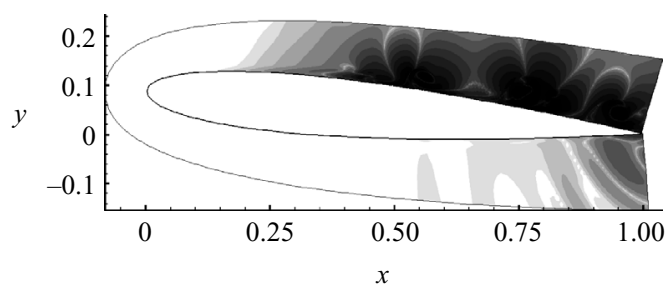

(b)

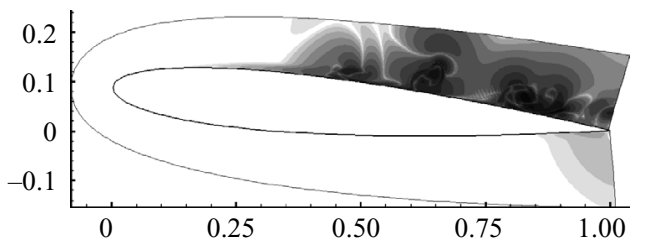

(d)

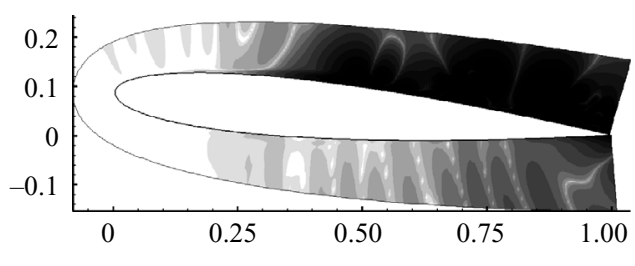

FIGURE 21. Iso-contours of $|w|$ velocity in the vicinity of the airfoil, using 20 contours exponentially distributed over the range $10^{-10}$ to $10^{-2}$, showing development with time after initialization. (a) $t=0.49$, (b) $t=0.98,(c) t=1.47$, (d) $t=1.96$.

to determine whether any instability mechanism is present in the current case that is not predicted by classical linear stability theory.

\subsection{Numerical method}

A three-dimensional simulation is initialized in the same manner as case 3DF. Grid G3 is used, specifying a small number of spanwise points (16) over the same spanwise domain width $\left(L_{z}=0.2\right)$. No time-periodic forcing is added, but $w$-perturbations are superposed onto the initial condition in the form of white noise. The $w$-perturbations are $1 \times 10^{-8}$ in amplitude, and only the boundary layer over the upper surface of the airfoil is seeded in this fashion. The simulation is progressed from this initial condition and no further disturbances are added. The stability characteristics of the unsteady two-dimensional separation bubble with vortex shedding may then be determined. The perturbations will either convect downstream whilst decaying or amplifying, ultimately leaving the flow over the airfoil unperturbed, or the perturbations will grow temporally without convecting downstream as in absolute instability. Effectively the method may be considered equivalent to a Floquet analysis; however the current method differs in that the base flow is not perfectly periodic in time.

\subsection{Time-dependent behaviour}

The resultant behaviour is illustrated by plotting absolute values of $w$-velocity in the vicinity of the airfoil at intervals of $t=0.49$ (figure 21). It can be seen that the initial disturbances do not convect downstream leaving the source unperturbed, but grow in amplitude temporally until nonlinear magnitudes are reached. The $w$-perturbations grow in amplitude within individual vortices as they convect downstream; however, within the vicinity of the vortex shedding location the perturbations also exhibit growth in amplitude without convecting downstream. Temporal growth occurs immediately upon initialization, and hence the onset is far too rapid to be explained by an acoustic feedback mechanism involving the trailing edge. The $N$-factor across the separated region has been computed via linear stability analysis to be $N=9.5$. This 


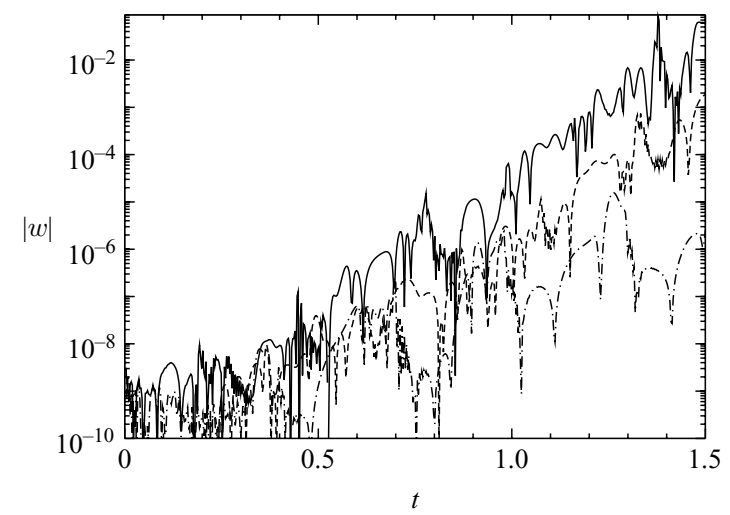

FIGURE 22. Time series of absolute $w$-velocity taken in the vicinity of vortex shedding at $5^{\circ}(-\cdot), 7^{\circ}(--)$ and $8.5^{\circ}(-)$ incidence.

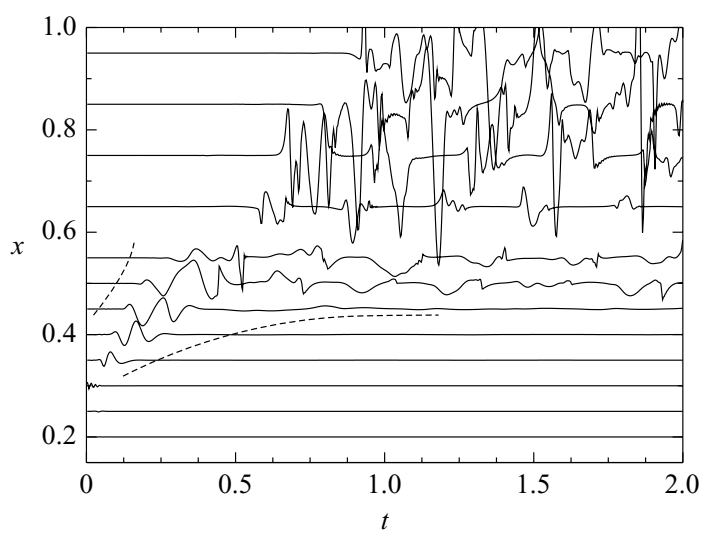

FIGURE 23. Time series of $w$ scaled by multiplying with $\mathrm{e}^{-4 t}$, taken at several locations within the boundary layer; the dashed lines indicate the wave-packet envelope.

precludes amplification of round-off error as a route to transition, since a much larger $N$-factor is required to amplify round-off error $\left(\sim 10^{-16}\right)$ to nonlinear amplitudes.

Having observed rapid temporal perturbation growth for the case with twodimensional vortex shedding at $5^{\circ}$ incidence, the simulation was repeated at other angles of attack. The simulations all exhibited two-dimensional vortex shedding. Absolute values of $w$-velocity taken in the vicinity of the onset of vortex shedding are plotted in figure 22. Although erratic due to variations during the shedding cycle, the amplitude of $w$-velocity perturbations appears to grow exponentially with time, and the temporal growth rate increases with incidence.

\subsection{Spatial onset}

In order to isolate the spatial onset of the instability, a simulation was run using a different initial disturbance input, and increased numbers of $w$-probes. Instead of seeding the entire upper airfoil boundary with white noise, a narrow 'strip' of white noise is used, located at $(x, y)=(0.25,0.136)$, and spanning the width of the domain. The evolution of the initial disturbance may then be tracked both spatially and temporally. Figure 23 shows an $x / t$ plot of disturbance growth for this case. Owing to the large growth rates present the probe readings were multiplied by $\mathrm{e}^{-\sigma t}$, where 
$\sigma=4$ is the temporal growth rate observed in the vicinity of vortex shedding, in order to better visualize the data. Therefore, where a probe signal appears constant in amplitude in figure 23, it is in fact growing at the rate of $\mathrm{e}^{4 t}$. The response to the perturbation varies with the $x$-location, as follows:

(i) For $x \leqslant 0.2$ no perturbations are observed using this scaling.

(ii) For $0.3 \leqslant x \leqslant 0.45$ the initial pulse generates a wavepacket in the boundary layer, which convects downstream. After $t=0.3$ no further disturbances are visible for $x \leqslant 0.4$ using this scaling.

(iii) For $0.5 \leqslant x \leqslant 0.55$ the initial wavepacket is observed to trigger disturbances that are lower in frequency than the initial disturbance, and are subsequently observed to grow exponentially in time.

(iv) For $x \geqslant 0.6$ it is difficult to detect whether the initial wavepacket is present or not. Periodic disturbances are observed to occur, with a frequency the same as that of the vortex shedding $(f=3.37)$. The amplitude of disturbances at any fixed $x$-location appears to grow at the approximate rate $\mathrm{e}^{4 t}$, and the amplitude of disturbances also appears to increase with increasing $x$-location.

Rapid, sustained temporal disturbance growth first occurs in the region $0.5 \leqslant x \leqslant 0.55$, suggesting that some form of absolute instability is sustained in the vicinity of the vortex shedding region. The temporal growth rate of perturbations appears approximately constant at all locations $(\sigma \approx 4)$; however the increase in amplitude of perturbations with $x$-location for $x \geqslant 0.6$ suggests that the flow is also convectively unstable.

\subsection{Instability mechanism}

The preceding section has identified a region of absolute instability in the vicinity of the vortex shedding location. Because the instability is subject to exponential growth, plots of perturbation quantities such as streamwise vorticity, $\omega_{x}$, or $w$-velocity, for example, will vary markedly in amplitude depending on the time at which they are taken. To surmount this problem, the quantity $\omega_{x}^{*}$ is plotted instead, defined as

$$
\omega_{x}^{*}=\omega_{x} A_{0} \mathrm{e}^{-\sigma t},
$$

where $A_{0}$ is a constant chosen as $1 \times 10^{-8}$ and $\sigma=4$ is the exponential disturbance growth rate at $x=0.5$. Plotting iso-surfaces of $\omega_{x}^{*}$ in the vicinity of vortex shedding at five phases, $\phi$, of the shedding cycle (figure 24) reveals spanwise-periodic structures that are associated with the absolute instability. Depending on the phase of the vortex cycle, the structures appear both within and also wrapped around the spanwise vortices. Although only one shedding cycle is illustrated, the behaviour of the instability appears qualitatively similar from one cycle to the next.

The production and behaviour of $\omega_{x}$ is illustrated more clearly in figure 25, again for five phases within the shedding cycle. Images on the left of figure 25 illustrate the two-dimensional vortex shedding upon which the three-dimensional perturbations are growing, as well as regions of upstream fluid flow. Images on the right of figure 25 illustrate the spanwise root-mean-square (RMS) of $\omega_{x}^{*}$, and hence indicate the magnitude of three-dimensional perturbations. Over the course of the shedding cycle a vortex is generated at the rear of the separation bubble before being released downstream. As the vortex begins to convect downstream, the magnitude of $\omega_{x}$ increases within the vortex core at a rate faster than the overall instability growth rate, as indicated by the increase in $\omega_{x}^{*}$ from figures $25(a)$ to 25(e). A second region of $\omega_{x}^{*}$ growth is also observed during the shedding cycle, just upstream of the developing vortex in the so-called 'braid' region. At $\phi=\frac{4}{5} \pi$ the region 

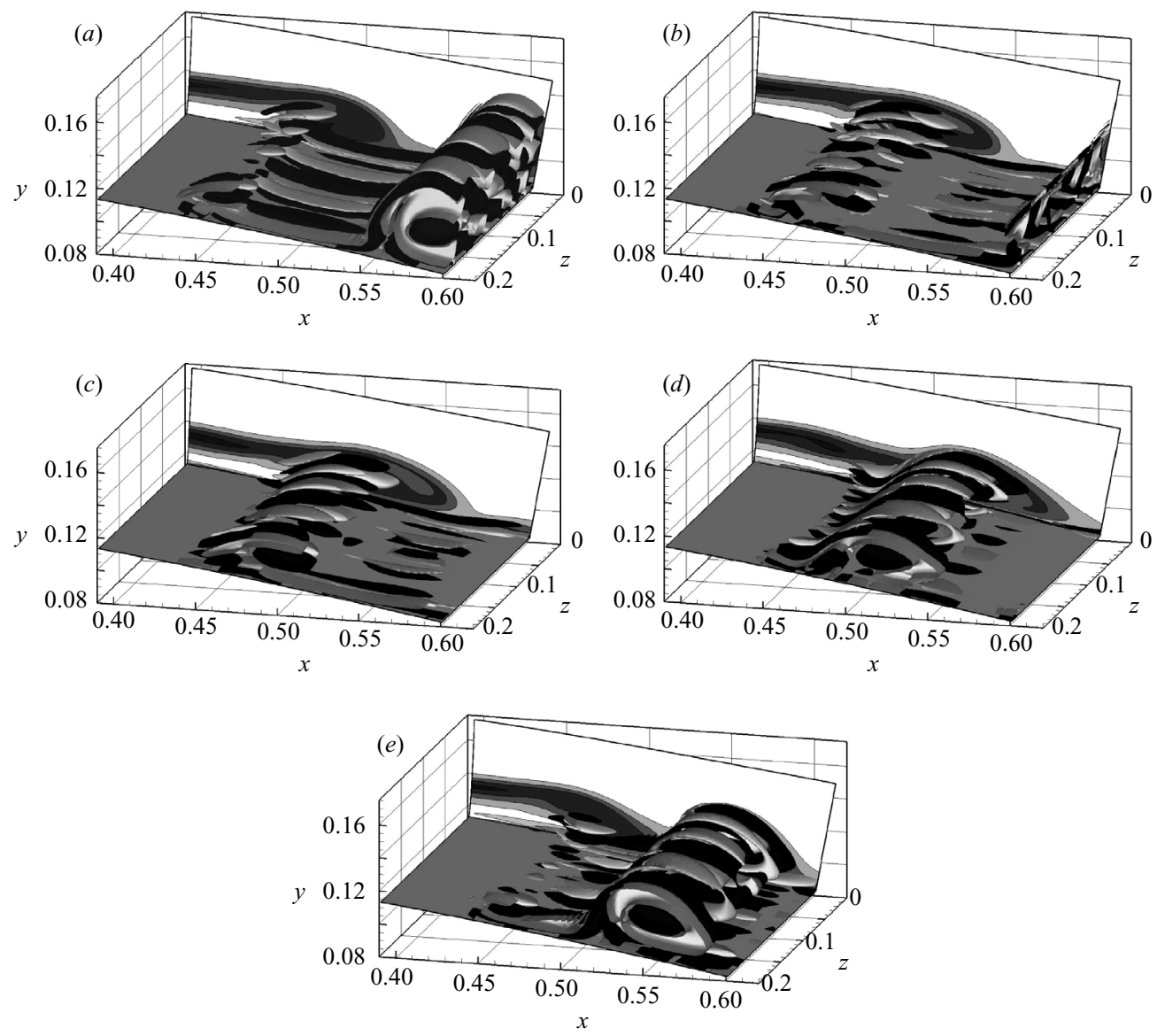

FIGURE 24. Iso-surfaces of $\omega_{x}^{*}$ taken at five phases of the vortex shedding cycle: $(a) \phi=0$, (b) $\phi=2 \pi / 5,(c) \phi=4 \pi / 5,(d) \phi=6 \pi / 5$, (e) $\phi=8 \pi / 5$. The far $(x, y)$-plane displays iso-contours of $\omega_{z}$, using ten levels over the range \pm 150 .

exhibiting the largest magnitude of $\omega_{x}$ is orientated approximately parallel to the $x$ axis at $(x, y)=(0.485,0.125)$. As the developing vortex is shed and begins to convect downstream, this structure increases in length and vorticity magnitude and is wrapped around the upstream and downstream vortices, forming an S-shape visible at $\phi=\frac{8}{5} \pi$ just upstream of the downstream-travelling vortex. Again, $\omega_{x}$ increases at a rate faster than the overall instability growth rate. These regions of pronounced perturbation growth appear to closely match regions of instability growth identified in mixing layers and bluff-body wakes, namely the vortex cores and the braid region between vortices (Williamson 1996). The current case appears to have a stronger analogy to shear-layer flow; however the mechanisms responsible for instability growth in braid regions and vortex cores are more extensively discussed in the literature for bluff-body wakes. In the context of bluff body-wakes, short-wavelength perturbations within vortex cores are commonly attributed to elliptic instability, whereas two forms of instability growth have been observed within the braid region, denoted mode-A and mode-B. A brief summary of each mode follows, necessary to categorize observations of the current case. 
(a)
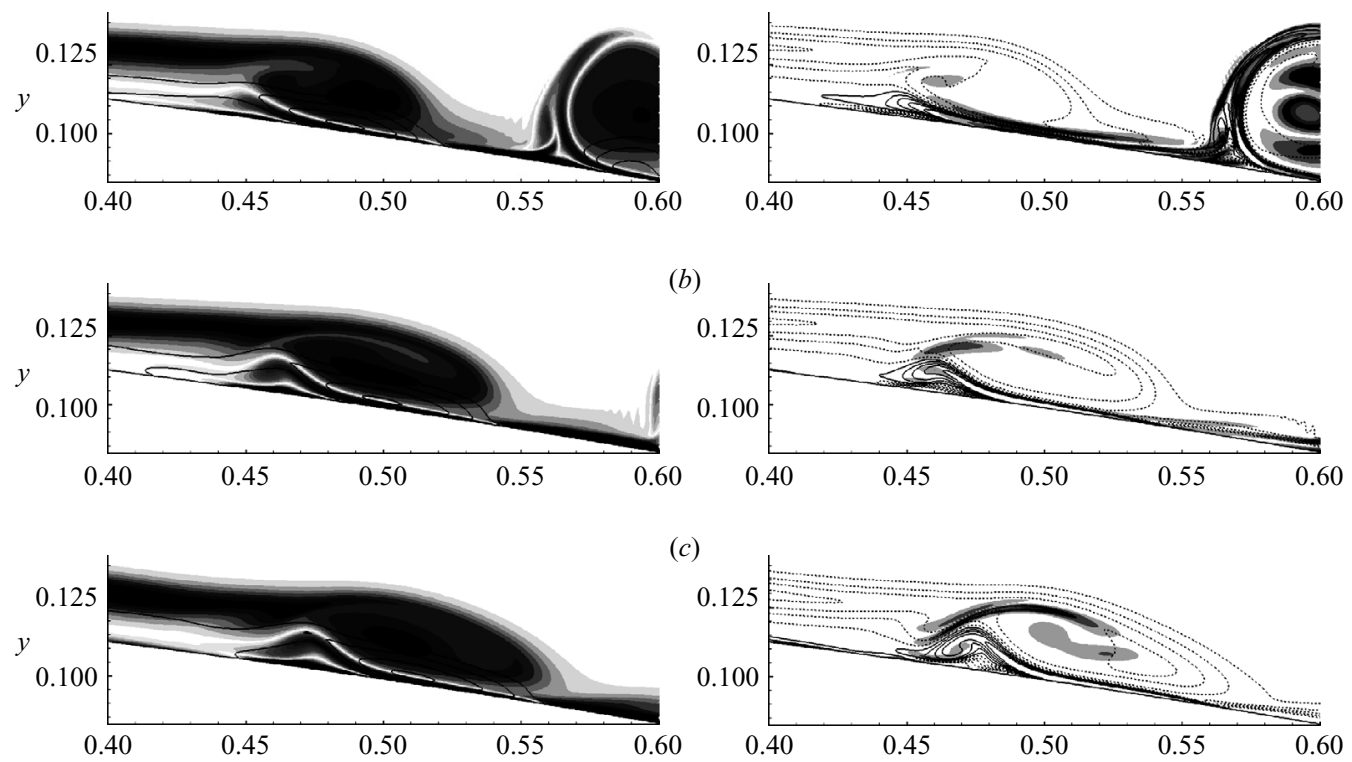

(d)
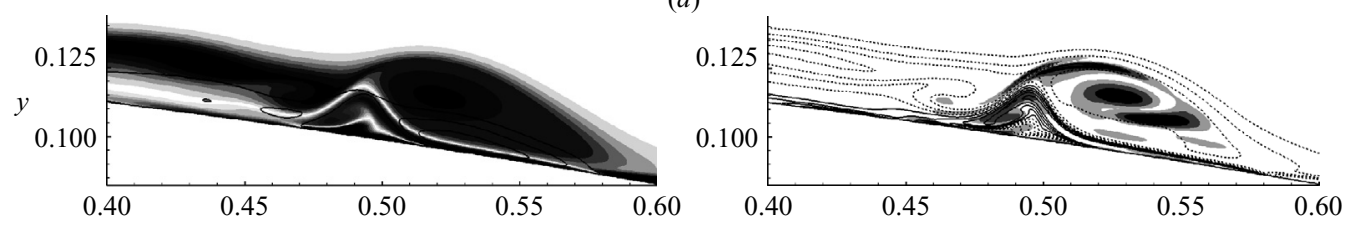

(e)
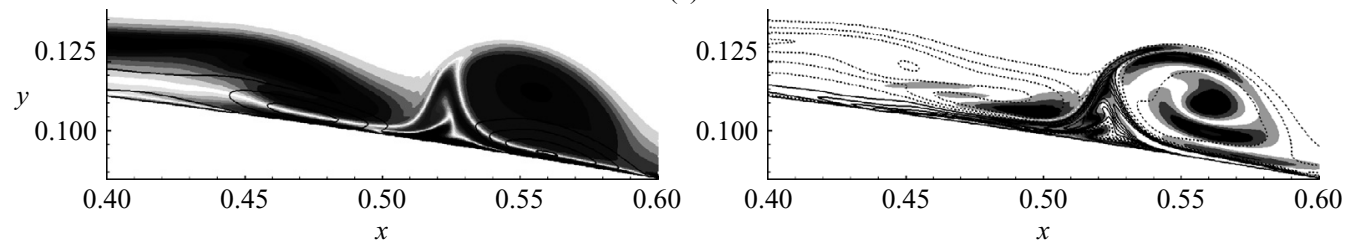

FIGURE 25. Left-hand images show iso-contours of $\omega_{z}$, using 20 contours over the range \pm 150 , with lines of constant $u$-velocity superposed using 4 levels over the range $-0.7<u<0$. Right-hand images show iso-contours of $\omega_{x}^{*}$ using 20 levels over the range 5-100 with lines of constant $\omega_{z}$ superposed using 10 levels over the range \pm 150 . Five phases within the vortex shedding cycle are shown: $(a) \phi=0,(b) \phi=2 \pi / 5,(c) \phi=\overline{4} \pi / 5,(d) \phi=6 \pi / 5,(e) \phi=8 \pi / 5$.

Elliptic instability is the name given to the instability of elliptical two-dimensional streamlines to three-dimensional perturbations, for which a review is given in Kerswell (2002). The physical mechanism of instability is vortex stretching, and the instability manifests itself as a spanwise-periodic deformation of the vortex core. Leweke \& Williamson (1998b) suggest that the spanwise wavelength of the most amplified instability mode is of the order $\lambda=3 D$, where $D$ is the diameter of the region of elliptical flow, comparing well with the results of Leweke \& Williamson (1998a). Floquet analysis by Barkley \& Henderson (1996) suggests a spanwise wavelength of $\lambda=4 D$ at onset. The presence of elliptic streamlines in the current case (figure 26) suggest that elliptic instability is likely to occur. 


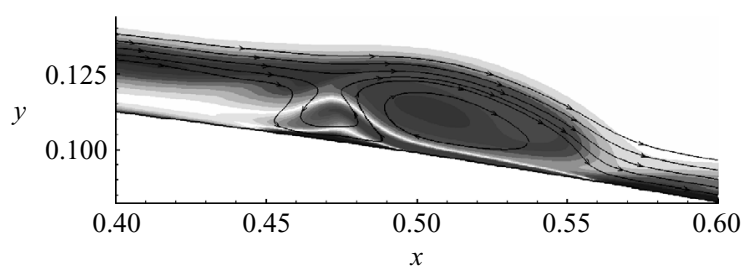

FIGURE 26. Iso-contours of $\omega_{z}$ taken at $\phi=4 \pi / 5$, using 20 levels over the range \pm 150 , with streamlines superposed illustrating both the presence of both hyperbolic streamlines upstream of a developing vortex and elliptic streamlines within the vortex itself.

Instability growth within the braid region between vortices has been observed experimentally, both for bluff-body wakes Williamson (1992) and free shear layers (Bernal \& Roshko 1986), as well as being studied numerically by Corcos \& Lin (1984). In bluff-body wakes, two distinct instabilities have been observed in the braid region, denoted mode-A and mode-B (Williamson 1996). Both forms of instability occur as spanwise-periodic streamwise 'tubes' of vorticity, formed in the braid region and extending between neighbouring two-dimensional vortices, that appear qualitatively similar to structures observed in figure 24. Mode-A instability is associated with spanwise wavelength approximately the same as that of elliptic instability, i.e. $3 D$ to $4 D$, and occurs in conjunction with deformation of the vortex core, whereas mode- $\mathrm{B}$ is associated with spanwise wavelength approximately $\lambda=D$ and occurs with no deformation of the vortex core (Williamson 1996). In the light of these differences it has been suggested that mode-A is caused by elliptic instability (Thompson, Leweke $\&$ Williamson 2001), and that mode-B is in fact a manifestation of the instability of two-dimensional hyperbolic streamlines, analogous to that of elliptic instability (Leweke \& Williamson 1998b), denoted hyperbolic instability. For bluff-body wakes mode-A is first observed at $R e_{d}>190$, where $R e_{d}$ is the Reynolds number based on cylinder diameter, and mode-B is first observed at $R e_{d}>240$.

Having identified similar regions of instability growth to those observed in bluffbody vortex shedding, the spanwise wavelength of three-dimensional perturbations can be compared. The diameter, $D$, of vortices in the current case is approximately 0.05 . The corresponding spanwise wavelengths for elliptic and mode- $\mathrm{A}$ instability are therefore expected to be in the range $0.15<\lambda<0.2$, and the corresponding wavelength for mode-B instability is expected to be of the order $\lambda=0.05$. In figure 24 the most prominent structures present in braid regions are streaks of $\omega_{x}$, which possess a spanwise wavelength of on average $\lambda=0.05$. This wavelength appears to correspond to that expected for mode-B instability, and is too small to be associated with either elliptic or mode-A instability. The spatial distribution of Floquet modes associated with mode-B instability in the study of Barkley \& Henderson (1996) appears to closely match regions outside vortex cores where growth is observed in the current case; hence the streamwise vortices produced in braid regions appear similar in nature to mode-B instability observed in bluff-body wakes. Streamlines plotted at $\phi=\frac{4}{5} \pi$ (figure 26) illustrate the presence of hyperbolic flow in the braid region upstream of each developing vortex, associated with a stagnation point, illustrating that the instability occurs in a region of hyperbolic flow, as suggested by Leweke \& Williamson (1998b) for mode-B instability.

In order to detect any elliptic instability, perturbations within the vortex cores must be analysed. It is difficult to observe the vortex cores in three-dimensional plots, since they are masked by the $\omega_{x}$ structures wrapped around the vortices. 


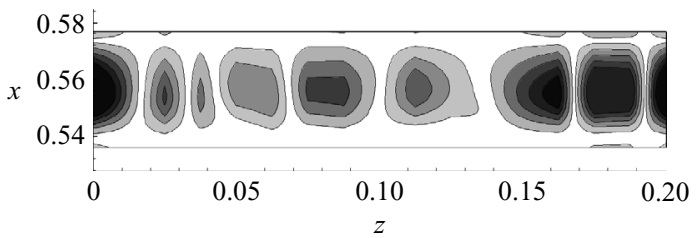

FIGURE 27. Iso-contours of $\omega_{x}$ taken across the centre of the vortex at $\phi=8 \pi / 5$, using 10 levels over the range \pm 100 .

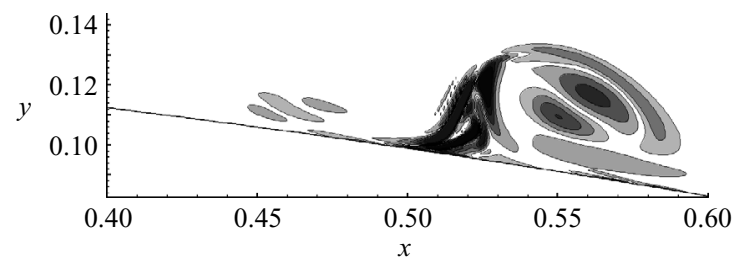

FIGURE 28. Iso-contours of perturbation $z$-vorticity at $t=1.96$ after initialization, corresponding to $\phi=8 \pi / 5$, using 10 levels over the range $\pm 5 \times 10^{-3}$.

Instead, iso-contours of $\omega_{x}^{*}$ are plotted for an $(x, z)$-plane through the vortex core at $\phi=\frac{8}{5} \pi$ in figure 27 . The vortex core exhibits pronounced perturbations with the same spanwise wavelength as observed outside the vortex core, i.e. 0.05. However, in contrast to perturbations outside the core which are of uniform amplitude across the span, $\omega_{x}^{*}$ is much larger in magnitude over the range $0.16<z<0.2\left(\omega_{x}^{*} \approx 100\right)$ than at $0.05<z<0.1\left(\omega_{x}^{*} \approx 50\right)$. Contours of perturbation $z$-vorticity taken at mid-span for $\phi=\frac{8}{5} \pi$, formed by subtracting the span-averaged $z$-vorticity from the instantaneous $z$-vorticity, are illustrated in figure 28. The structure within the vortex core appears similar to the localized perturbation solutions presented by Waleffe (1990) for unbounded elliptical flow, and is orientated along the axis of strain associated with the vortex deformation. The vortex core therefore appears perturbed at two distinct spanwise wavelengths with similar amplitude, $\lambda_{1} \approx 0.05$ and $\lambda_{2} \approx 0.2$. The first wavelength corresponds to that exhibited by $\omega_{x}$ structures outside the vortex core, i.e. mode-B instability; however the second wavelength is significantly larger and is comparable to wavelengths associated with elliptic instability. The structure of the perturbation within the vortex core appears similar to that associated with elliptic instability.

Evidence suggests then, that the production of $\omega_{x}$ occurs due to a combination of instabilities within the vortex cores and braid regions, appearing similar to elliptic and mode-B instabilities respectively, as observed in bluff-body wakes (Williamson 1996). It seems that the combination of elliptic and mode-A/B instabilities has not been considered in terms of its absolute or convective nature for bluff-body wakes. In the current case, although the instability mechanism differs from the classical definition of absolute instability for disturbances on parallel base flows, at a given $x$-location exponential temporal growth occurs. Hence the term absolute instability is still useful to describe the behaviour present. Effectively the mechanism acts as an 'oscillator' as opposed to an 'amplifier'.

The absolute nature of this instability can be explained by referring back to figure 25. At $\phi=\frac{8}{5} \pi$, in the region of hyperbolic flow upstream of the vortex a 


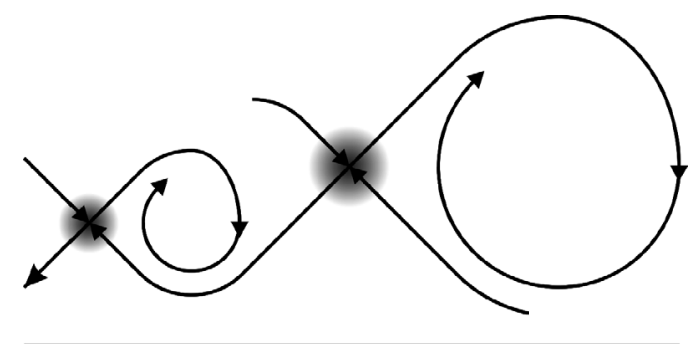

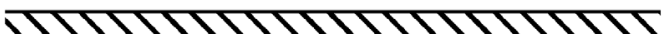

FIGURE 29. Schematic of the manner in which fluid exiting the braid region developed behind one vortex, subject to mode-B instability growth, enters the braid region associated with the subsequent vortex. Hyperbolic regions of fluid flow, where mode-B instability is observed to occur, are shaded.

comparatively long S-shaped perturbation with large $\omega_{x}$ magnitude is observed, attributed to the presence of mode-B instability. This perturbation extends into a region of strong upstream fluid flow $(u \approx-0.7)$ as illustrated in figure 25(e), left. Hence $\omega_{x}$ perturbations present in the braid region, amplified in the vicinity of hyperbolic flow, are convected upstream with comparatively large velocity and, critically, into the braid region associated with the next developing vortex. This can be clearly seen in figures $25(a)$ to $25(c)$. The long thin $\omega_{x}$ perturbation near the airfoil surface in the region $0.45<x<0.55$ in figure $25(a)$, which is a remnant of the previous shedding cycle, convects into a region of hyperbolic flow and generates the streamwise-orientated structure clearly visible in figure 25(c). This behaviour is also illustrated in three dimensions in figure 24. Clearly the absolute mechanism is driven by instability growth within the braid region of vortices. Instability growth within vortex cores, whilst exhibiting similar temporal growth rate, appears to exhibit little direct upstream influence.

A schematic for the absolute instability mechanism is given in figure 29 . Perturbations are amplified in braid regions, forming streamwise vortices, and extend into regions of high-magnitude reverse flow. These streamwise vortices are convected upstream and into the braid region of the next developing spanwise vortex. The process then repeats with increasing amplitude. Temporal growth is sustained owing to the existence of local regions of reverse flow for which the velocity magnitude greatly exceeds that of the time-average, in addition to large instability growth rates observed in hyperbolic regions of fluid flow. This transition mechanism is clearly not predictable via linear stability analysis of the time-averaged flow field, since it is a secondary instability of an unsteady behaviour. Case 3DF may thus be described as exhibiting transition driven by convective instability and case 3DU by absolute instability of two-dimensional vortex shedding, by a combination of instabilities similar to elliptic and mode-B instability observed in bluff-body wakes. There is clear evidence that the secondary absolute instability of a forced separation bubble observed by Maucher et al. (1998) is driven by the same mechanism; the behaviour observed by Maucher et al. appears similar in many respects, including similar transitional structures. The instability mechanism may also be responsible for the rapid breakdown to turbulence observed by Spalart \& Strelets (2000) in the absence of added disturbances. Laminar reattachment is not possible for the current case, owing to the presence of this absolute instability. 


\section{Conclusions}

DNS were conducted of a laminar separation bubble on a NACA-0012 airfoil at $5^{\circ}$ incidence. Both the three-dimensional separation bubble behaviour and the aerodynamic performance of the airfoil were found to be dependent on the presence of forcing. Compared to the unforced case, the inclusion of forcing increases the lift-to-drag ratio by approximately $23 \%$ and the intensity of turbulent/unsteady fluctuations over the airfoil is significantly reduced. Fluid structures downstream of transition are found to exhibit increased spanwise coherence in the unforced case. Forcing in a similar fashion could therefore potentially be used as a control mechanism for improving low-Reynolds-number airfoil performance. Both of the three-dimensional separation bubbles exhibited large temporal variation of skin friction. At no point downstream of transition was the flow either fully attached or fully separated, suggesting that the concept of a reattachment 'point' is misleading. Comparison of skin-friction PDFs illustrates that time-dependent behaviour of the two-dimensional separation bubble is fundamentally different to that of the threedimensional bubbles.

Classical linear stability analysis of the time-averaged flow fields suggests that the separation bubble is convectively unstable in all cases, since no evidence of absolute instability is observed. Upon the removal of forcing, however, the turbulence over the aft section of the airfoil is observed to self-sustain. A series of three-dimensional simulations, resolving the linear response to three-dimensional perturbations, suggest that the two-dimensional vortex shedding behaviour is absolutely unstable to threedimensional perturbations. The instability is associated with the production of streamwise vortices located in the braid region between successive spanwise vortices, with spanwise wavenumber comparable to that of mode-B instability as observed in bluff-body wakes. A mechanism by which the instability can self-sustain is proposed, dependent on strong local reverse flow and large instability growth rates in braid regions. The temporal growth rate of the instability increases with airfoil incidence, presumably owing to the larger magnitude of reverse flow present in the twodimensional separation bubble.

It appears therefore, that in the absence of convectively driven transition within the shear layer, transition will take place by absolute instability of the two-dimensional vortex shedding in a manner not predicted by linear stability analysis of the timeaveraged flowfield. This has important implications for the modelling of laminar separation bubbles, suggesting that if free-stream turbulence levels drop below a certain value, the time-averaged transition and reattachment locations will be fixed and not vary with further decreases in free-stream turbulence levels.

Computer time was provided by the EPSRC grant GR/S27474/01.

\section{REFERENCES}

Alam, M. \& Sandham, N. D. 2000 Direct numerical simulation of shortlaminar separation bubbles with turbulent reattachment. J. Fluid Mech. 410, 1-28.

Barkley, D. \& Henderson, R. D. 1996 Three-dimensional Floquet stability analysis of the wake of a circular cylinder. J. Fluid Mech. 322, 215-241.

Bernal, L. P. \& Roshko, A. 1986 Streamwise vortex structure in plane mixing layers. J. Fluid Mech. 170, 499-525.

Carpenter, M. H., Nordström, J. \& Gottlleb, D. 1999 A stable and conservative interface treatment of arbitrary spatial accuracy. J. Comput. Phys. 148, 341-365. 
Corcos, G. M. \& Lin, S. J. 1984 The mixing layer: Deterministic models of a turbulent flow. Part 2. The origin of the three-dimensional motion. J. Fluid Mech. 139, 67-95.

Drazin, P. G. \& ReED, W. H. 1981 Hydrodynamic Stability. Cambridge University Press.

Drela, M. \& Giles, M. B. 1987 Viscous-inviscid analysis of transonic and low Reynolds number airfoils. AIAA J. 25, 1347-1355.

Gaster, M. 1967 The structure and behaviour of separation bubbles. Aero. Res. Counc. R\&M 3595. Aerodynamics Division NPL.

GASTER, M. 1978 Series representation of the eigenvalues of the Orr-Sommerfeld equation. J. Comput. Phys. 29, 147-162.

GaUlt, D. E. 1957 A correlation of low-speed airfoil-section stalling characteristics with Reynolds number and airfoil geometry. NACA TN 3963. Washington.

Hammond, D. A. \& Redekopp, L. G. 1998 Local and global instability properties of separation bubbles. Eur. J. Mech. B/Fluids 17, 145-164.

Hannemann, K. \& Oertel, H. 1989 Numerical simulation of the absolutely and convectively unstable wake. J. Fluid Mech. 199, 55-88.

Horton, H. P. 1969 A semi-empirical theory for the growth and bursting of laminar separation bubbles. Aero. Res. Counc. Current Paper 1073.

Hu, Z. W., Morfey, C. L. \& Sandham, N. D. 2006 Wall pressure and shear stress spectra from direct simulations of channel flow. AIAA J. 44, 1541-1549.

Huerre, P. \& Monkewitz, P. A. 1985 Absolute and convective instabilities in free shear layers. J. Fluid Mech. 159, 151-168.

Kerho, M., Hutcherson, S., Blackwelder, R. F. \& Liebeck, R. H. 1993 Vortex generators used to control laminar separation bubbles. J. Aircraft 30, 315-319.

Kerswell, R. R. 2002 Elliptical Instability. Annu. Rev. Fluid Mech. 34, 83-113.

LANG, M., RIST, U. \& WAGNER, S. 2004 Investigations on controlled transition development in a laminar separation bubble by means of LDA and PIV. Exps. Fluids 36, 43-52.

Lele, S. K. 1992 Compact finite difference schemes with spectral-like resolution. J. Comput. Phys. $103,16-42$.

Leweke, T. \& Williamson, C. H. K. 1998 a Cooperative elliptic instability of a vortex pair. J. Fluid Mech. 360, 85-119.

Leweke, T. \& Williamson, C. H. K. $1998 b$ Three-dimensional instabilities in wake transition. Eur. J. Mech. B/Fluids 17, 571-586.

MarXen, O., Lang, M., Rist, U. \& Wagner, S. 2003 A combined experimental/numerical study of unsteady phenomena in a laminar separation bubble. Flow, Turbulence Combust. 71, 133146.

Marxen, O., Rist, U. \& Wagner, S. 2004 Effect of spanwise-modulated disturbances on transition in a separated boundary layer. AIAA J. 42, 937-944.

Maucher, U., Rist, U. \& Wagner, S. 1997 Secondary instabilities in a laminar separation bubble. In New Results in Numerical and Experimental Fluid Dynamics (ed. H. Körner \& R. Hilbig), pp. 229-236. Vieweg.

MAUCHER, U., Rist, U. \& WAGNeR, S. 1998 Transitional structures in a laminar separation bubble. New Results in Numerical and Experimental Fluid Mechanics II, Proc. 11th STAB/DGLR Symposium, Berlin (ed. W. Nitsche, H. J. Heinemann \& R. Hilbig), vol. 72, pp. 307-314.

PAuley, L. L. 1994 Response of two-dimensional separation to three-dimensional disturbances. Trans. ASME: J. Fluids Engng 116, 433-438.

Pauley, L. L., Moin, P. \& Reynolds, W. C. 1990 The structure of two-dimensional separation. J. Fluid Mech. 220, 397-411.

Rist, U. 1994 Nonlinear effects of 2D and 3D disturbances on laminar separation bubbles. Proc. IUTAM Symp. on Nonlinear Instability of Nonparallel Flows, pp. 324-333. Springer.

Rist, U. \& MAUCHER, U. 2002 Investigations of time-growing instabilities in laminar separation bubbles. Eur. J. Mech. B/Fluids 21, 495-509.

SANDBERG, R. D. \& SANDhAm, N. D. 2006 Nonreflecting zonal characteristic boundary condition for direct numerical simulation of aerodynamic sound. AIAA J. 44, 402-405.

Sandberg, R. D., Sandham, N. D. \& Joseph, P. F. 2007 Direct numerical simulations of trailing-edge noise generated by boundary-layer instabilities. J. Sound Vib. 304, 677-690.

Sandham, N. D., LI, Q. \& YeE, H. C. 2002 Entropy splitting for high-order numerical simulation of compressible turbulence. J. Comput. Phys. 178, 307-322. 
Sandhu, H. S. \& Sandham, N. D. 1994 Boundary conditions for spatially growing compressible shear layers. Rep. QMW-EP-1100. Faculty of Engineering, Queen Mary and Westfield College, University of London.

Schmid, P. J. \& Henningson, D. S. 2001 Stability and Transition in Shear Flows. Springer.

Spalart, P. R. \& Strelets, M. K. H. 2000 Mechanisms of transition and heat transfer in a separation bubble. J. Fluid Mech. 403, 329-349.

SpalaRT, P. R. 1988 Direct simulation of a turbulent boundary layer up to $R_{\theta}=1410$. J. Fluid Mech. 187, 61-98.

VON TERZI, D. A. 2004 Numerical investigation of transitional and turbulent backward-facing step flows. PhD thesis, The University of Arizona.

Theofilis, V. 2000 On the origins of unsteadiness and three-dimensionality in a laminar separation bubble. Phil. Trans. R. Soc. Lond. 358, 3229-3246.

Theofilis, V. 2003 Advances in global linear instability analysis of nonparallel and three-dimensional flows. Prog. Aerospace Sci. 39, 249-315.

Thompson, M. C., LeweKe, T. \& Williamson, C. H. K. 2001 The physical mechanism of transition in bluff body wakes. J. Fluids Struct. 15, 607-616.

WalefFe, F. 1990 On the three-dimensional instability of strained vortices. Phys. Fluids A 2, 76.

Williamson, C. H. K. 1992 The natural and forced formation of spot-like'vortex dislocations' in the transition of a wake. J. Fluid Mech. 243, 393-441.

Williamson, C. H. K. 1996 Three-dimensional wake transition. J. Fluid Mech. 328, 345-407. 